\title{
Epithelial myosin light chain kinase- dependent barrier dysfunction mediates T cell activation-induced diarrhea in vivo
}

\author{
Daniel R. Clayburgh, ${ }^{1}$ Terrence A. Barrett, ${ }^{2}$ Yueming Tang, ${ }^{2}$ Jon B. Meddings, ${ }^{3}$ Linda J. Van Eldik, ${ }^{4}$ \\ D. Martin Watterson, ${ }^{4}$ Lane L. Clarke, ${ }^{5}$ Randall J. Mrsny, ${ }^{6}$ and Jerrold R. Turner ${ }^{1}$ \\ 1Department of Pathology, The University of Chicago, Chicago, Illinois, USA. ${ }^{2}$ Department of Medicine, Division of Gastroenterology, \\ Northwestern University, Chicago, Illinois, USA. ${ }^{3}$ Department of Medicine, University of Alberta, Edmonton, Alberta, Canada. \\ ${ }^{4}$ Center for Drug Discovery and Chemical Biology, Northwestern University, Chicago, Illinois, USA. ${ }^{5}$ Department of Biomedical Sciences, \\ University of Missouri, Columbia, Missouri, USA. ${ }^{6}$ Welsh School of Pharmacy, Cardiff University, Cardiff, Wales, United Kingdom.
}

\begin{abstract}
Disruption of the intestinal epithelial barrier occurs in many intestinal diseases, but neither the mechanisms nor the contribution of barrier dysfunction to disease pathogenesis have been defined. We utilized a murine model of $\mathrm{T}$ cell-mediated acute diarrhea to investigate the role of the epithelial barrier in diarrheal disease. We show that epithelial barrier dysfunction is required for the development of diarrhea. This diarrhea is characterized by reversal of net water flux, from absorption to secretion; increased leak of serum protein into the intestinal lumen; and altered tight junction structure. Phosphorylation of epithelial myosin II regulatory light chain (MLC), which has been correlated with tight junction regulation in vitro, increased abruptly after $T$ cell activation and coincided with the development of diarrhea. Genetic knockout of long myosin light chain kinase (MLCK) or treatment of wild-type mice with a highly specific peptide MLCK inhibitor prevented epithelial MLC phosphorylation, tight junction disruption, protein leak, and diarrhea following T cell activation. These data show that epithelial MLCK is essential for intestinal barrier dysfunction and that this barrier dysfunction is critical to pathogenesis of diarrheal disease. The data also indicate that inhibition of epithelial MLCK may be an effective non-immunosuppressive therapy for treatment of immune-mediated intestinal disease.
\end{abstract}

\section{Introduction}

The intestinal epithelium is positioned at a critical interface between the interstitium and the harsh environment of the gut lumen. Thus, the epithelium must form a selectively permeable barrier that allows absorption of nutrients, electrolytes, and water while preventing passage of noxious luminal contents. This barrier is disrupted in many diseases, including inflammatory bowel disease, graft versus host disease, and infectious enterocolitis (1-6). While some have suggested that this barrier dysfunction is a central component of disease pathogenesis (7-9), other work suggests that barrier dysfunction may be a secondary event that does not contribute to disease progression (10). Thus, the relevance of barrier dysfunction to intestinal disease pathogenesis remains controversial.

The primary explanation for the controversy regarding the role of barrier dysfunction in intestinal disease is the absence of specific means of restoring barrier function. In large part this lack of barrier-restoring agents reflects the paucity of information regarding in vivo mechanisms of barrier dysfunction. For example, it is clear that TNF can disrupt the epithelial barrier in vivo and in vitro $(2,8,11-15)$. Moreover, in vivo studies of humans with Crohn disease and mice with experimental graft versus host disease show

Nonstandard abbreviations used: CFTR, cystic fibrosis transmembrane conductance regulator; CMF-HBSS, $\mathrm{Ca}^{2+}$ - and $\mathrm{Mg}^{+}$-free HBSS; JAM-A, junctional adhesion molecule A; MLC, myosin II regulatory light chain; MLCK, myosin light chain kinase; $\mathrm{NHE}, \mathrm{Na}^{+}-\mathrm{H}^{+}$exchanger; $\mathrm{PIK}$, membrane-permeant inhibitor of MLCK; ZO-1, zonula occludens-1.

Conflict of interest: The authors have declared that no conflict of interest exists.

Citation for this article: J. Clin. Invest. 115:2702-2715 (2005).

doi:10.1172/JCI24970. that TNF antagonism results in restoration of intestinal barrier function $(2,13)$. However, it is not clear whether this restoration of barrier function is due to interruption of TNF signaling to the epithelium or, alternatively, reflects overall suppression of immune activation. Thus, although a correlation between mucosal cytokine levels and epithelial barrier function has been established, neither the mechanisms by which inflammatory processes disrupt in vivo intestinal barrier function nor the relationship between barrier disruption and disease evolution are understood.

In vitro studies have suggested a variety of mechanisms for cytokine-dependent tight junction disruption, including downregulation of tight junction protein transcription $(16,17)$, decreased $\mathrm{Na}^{+}, \mathrm{K}^{+}$-ATPase expression (18), epithelial apoptosis (19), and cytoskeletal contraction $(11,20,21)$. Of these, cytoskeletal contraction due to myosin II regulatory light chain (MLC) phosphorylation has been demonstrated to be necessary for barrier disruption, as in vitro inhibition of myosin light chain kinase (MLCK) restores barrier function after cytokine treatment $(11,20,21)$. A variety of in vitro analyses suggest that MLC phosphorylation and subsequent cytoskeletal contraction constitute a common mechanism of barrier regulation in response to physiological and pathophysiological stimuli, including nutrient transport (22) and parasitic and bacterial infection $(1,23)$, and that this epithelial MLC phosphorylation is primarily accomplished by long MLCK $(11,24)$. Despite these advances, in vitro studies of cultured monolayers do not recreate the complex interactions between epithelia and other cell types present in vivo nor do they provide insight into the development of actual disease symptoms such as diarrhea. Thus, a significant gap exists in our knowledge 
A

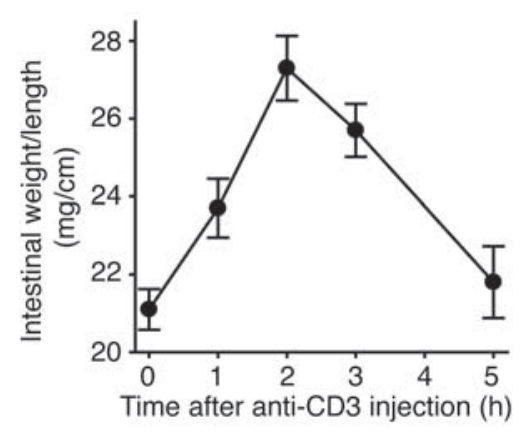

B

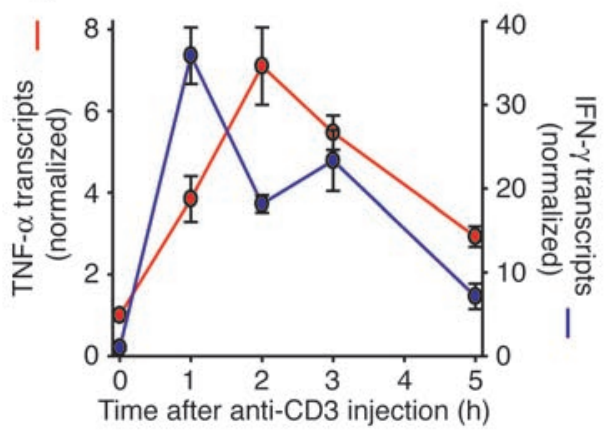

C

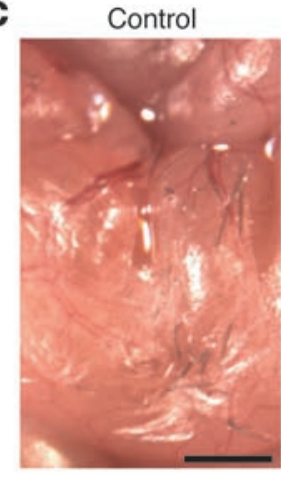

+ Anti-CD3

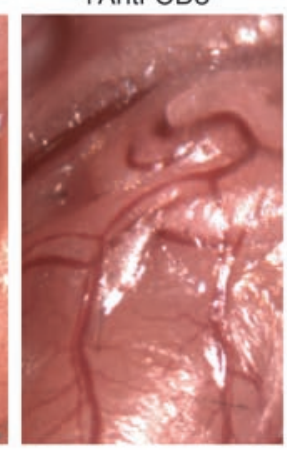

Figure 1

Anti-CD3 injection causes diarrhea associated with Th1 cytokine induction. (A) The weight-to-length ratio of the small intestine, an indicator of tissue edema and luminal fluid accumulation, was increased after anti-CD3 injection. The weight-to-length ratio peaked 2-3 hours after injection and returned to baseline within 5-6 hours after injection ( $n=4$ for each time point). (B) Mucosal IFN- $\gamma$ (blue line) and TNF- $\alpha$ (red line) transcription increased markedly after anti-CD3 injection, but returned to levels only slightly higher than baseline by 5 hours after injection ( $n=3$ for each time point). (C) Examination of the serosa in control and anti-CD3-treated mice revealed classic signs of inflammation after anti-CD3 injection, including vasodilation, edema, and erythema (scale bar, $0.5 \mathrm{~mm}$ ).

of the role and mechanisms of pathologic barrier dysfunction in the development of intestinal disease that cannot be adequately addressed by cell culture models.

To define the role of barrier dysfunction in disease pathogenesis, we sought an in vivo model of acute immune-mediated diarrheal disease. Systemic T cell activation induced by administration of antiCD3 antibodies causes acute diarrhea in humans and mice (25-28) that is associated with barrier dysfunction (14). This diarrhea is accompanied by induction of IFN- $\gamma$ and TNF- $\alpha$ and can be blocked by antagonism of either cytokine $(26,29)$. Thus, in addition to being related to the acute syndrome seen in human patients treated with $\mathrm{T}$ cell-activating OKT3 antibodies, this diarrhea may be mechanistically related to graft versus host disease and inflammatory bowel disease. We asked whether epithelial barrier dysfunction was necessary for progression of this model disease and if reversal of barrier dysfunction could lessen disease severity. The data show that MLCKmediated barrier dysfunction is required for diarrhea to occur after $\mathrm{T}$ cell activation and further suggest that MLCK may be a viable target for the treatment of immune-mediated diarrheal disease.

\section{Results}

Systemic T cell activation causes acute diarrhea independent of epithelial apoptosis. The systemic T cell activation that is induced by injection of anti-CD3 antibodies induces acute TNF-dependent diarrhea in humans and mice $(14,27,30)$. We quantified the development of this diarrhea in mice using the small intestinal weight-to-length ratio, a measure of luminal fluid accumulation and tissue edema (14). Increases in weight-to-length ratio followed a highly reproducible kinetic pattern peaking after 2 to 3 hours and resolving at 5 hours after intraperitoneal anti-CD3 injection (Figure 1A). Consistent with previous reports $(14,28,31)$, levels of mucosal IFN- $\gamma$ and TNF- $\alpha$ (Figure 1B) transcripts increased markedly after antiCD3 injection but returned toward baseline levels by 5 hours after injection. These anti-CD3-induced increases in weight-to-length ratio and cytokine induction were also accompanied by gross evidence of intestinal inflammation. The small intestinal serosa of anti-CD3-treated animals revealed classic signs of inflammation, including vasodilation, vascular injection, and edema (Figure 1C).
Thus anti-CD3 injection is an effective means of inducing an acute, self-limited, cytokine-mediated diarrhea in mice.

The proinflammatory cytokines produced after anti-CD3 injection, in particular TNF- $\alpha$, are potent stimulators of apoptotic cell death in many cell populations. Apoptotic death of epithelial cells has been postulated to be a factor in the TNF-dependent epithelial barrier dysfunction that occurs in vitro (19), although this idea is controversial, as other in vitro studies have shown that apoptosis is not involved in some models of cytokine-mediated barrier dysfunction $(11,15)$. Thus, we investigated in vivo epithelial apoptosis in anti-CD3-induced diarrhea. Macroscopic examination of the intestinal mucosa 3 hours after anti-CD3 injection demonstrated tissue edema, seen as a distortion of the normal mucosal folds (Figure 2A), but no gross ulceration or tissue damage that might be associated with massive epithelial apoptosis. Histological examination confirmed that 3 hours after anti-CD3 treatment, no ulceration or erosion was present (Figure $2 \mathrm{~B}$ ). Moreover, the ratio of villus to total villus-crypt length $(0.65 \pm 0.01$ in controls versus $0.66 \pm 0.01$ in anti-CD3-treated animals; $P=0.22$ ) was unchanged after anti-CD3 treatment. However anti-CD3 did cause morphologically evident villous thickening that could be quantified as an increase in villous width-to-height ratio $(0.24 \pm 0.01$ in controls versus $0.40 \pm 0.02$ after anti-CD3 treatment; $P<0.0001)$. This may reflect lamina propria edema. Anti-CD3 treatment also caused an increase in numbers of intraepithelial lymphocytes, from $5.1 \pm 0.2$ to $7.9 \pm 0.3$ per 100 epithelial cells 3 hours after anti-CD3 injection $(P<0.0001)$. However, anti-CD3 treatment did not induce an increase in the number of morphologically evident apoptotic epithelial cells (Figure 2, C and D). There was also no biochemical evidence of epithelial apoptosis. Immunoblots of lysates of epithelial cells isolated 3 hours after anti-CD3 injection revealed no increase in conversion of caspase- 3 to the cleaved (active) form (Figure 2E). Together, these data show that epithelial apoptosis is not increased in the first 3 hours after anti-CD3 injection, thus demonstrating that the associated diarrhea is not due to epithelial apoptosis.

Systemic T cell activation induces a TNF-dependent net reversal of water flow and leakage of serum proteins into the intestinal lumen. Changes in intestinal transport and barrier function induced by anti-CD3 


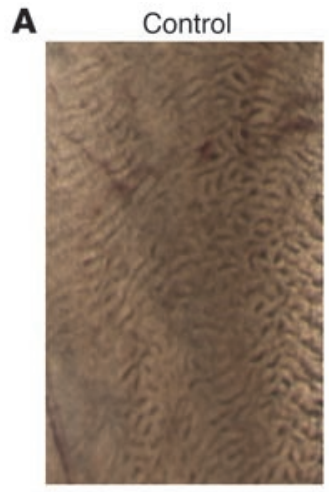

C
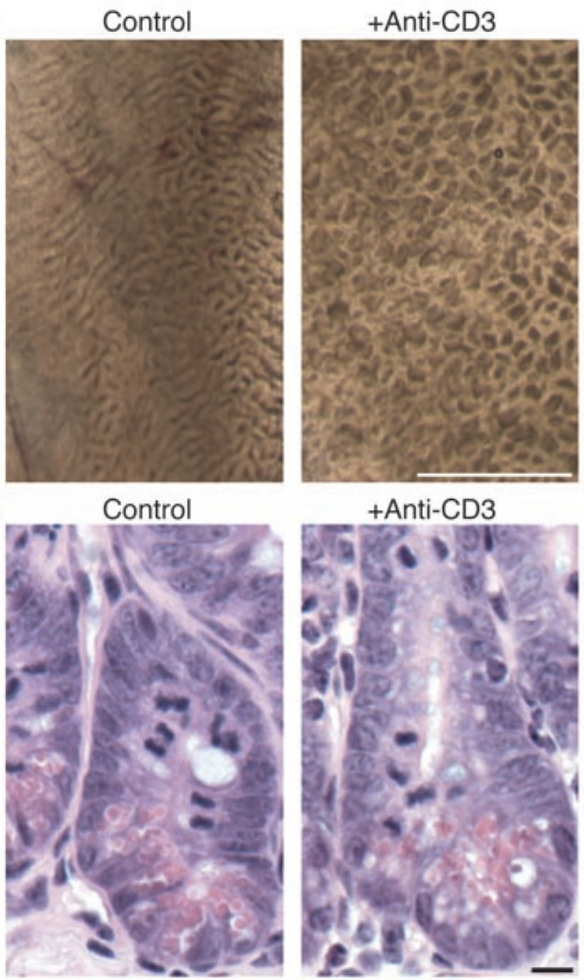

B

D

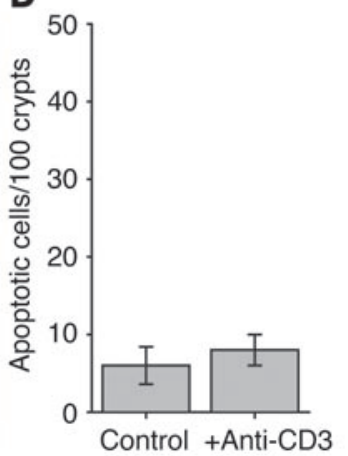

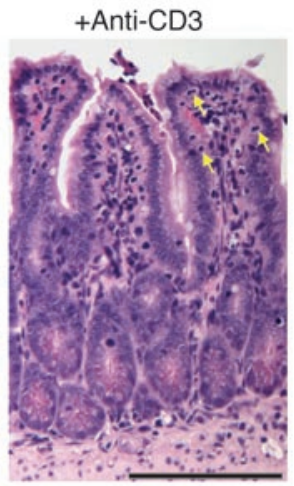

E

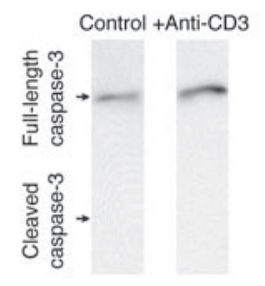

Figure 2

Anti-CD3-induced diarrhea is associated with edema and increased numbers of intraepithelial lymphocytes but not ulceration or epithelial apoptosis. (A) Macroscopic examination of the small intestinal mucosae 3 hours after anti-CD3 injection showed distortion of the normal mucosal folds by edema and luminal fluid accumulation. Notably, anti-CD3 injection did not result in mucosal ulceration or erosion (scale bar, $1 \mathrm{~mm}$ ). (B) Microscopic examination of the small intestinal mucosae revealed mild villous thickening 3 hours after anti-CD3 injection. An increase in the number of intraepithelial lymphocytes (arrows) was also present ( $5.1 \pm 0.2$ intraepithelial lymphocytes per 100 epithelial cells in control tissue vs. $7.9 \pm 0.3$ intraepithelial lymphocytes per 100 epithelial cells 3 hours after anti-CD3 injection; $P<0.0001$ ), but there was no apparent epithelial damage or ulceration (scale bar, $100 \mu \mathrm{m}$ ). (C) Closer examination of the crypts showed mitotic figures in both control and anti-CD3-treated jejunum, but apoptotic cells were rare both before and 3 hours after anti-CD3 treatment (scale bar, $10 \mu \mathrm{m}$ ). (D) Quantitative analysis of apoptotic epithelial cells revealed no significant increase in number 3 hours after anti-CD3 injection. (E) Immunoblots for caspase3 in isolated small intestinal epithelial cells 3 hours after vehicle or anti-CD3 injection detected only the uncleaved (inactive) form of this protein, indicating minimal caspase- 3 activation.

treatment were previously studied ex vivo using excised small intestine mounted in Üssing chambers $(14,30)$. However, this procedure requires the tissue to be isolated from the body, thus interrupting the neurovascular supply and removing important stimuli. Moreover, while Üssing chambers may be used to measure ion transport and barrier function, they are less useful for measuring net water movement, the hallmark of diarrhea. To measure water movement and barrier function in vivo after $\mathrm{T}$ cell activation, we adapted a method previously described for analysis of absorption in rats (32) for analysis of water movement and barrier function in mice (Figure 3A). In this in vivo system, a small loop of intestine in an anesthetized mouse is cannulated at both ends and perfused in a recirculating manner. Although the lumen is isolated from the rest of the alimentary tract, this method maintains the vasculature and innervation of the intestinal mucosa. The perfusion solution contains the highly charged anion ferrocyanide, which is neither absorbed nor secreted and can therefore be used to monitor the movement of water into or out of the perfused intestinal segment. (33-35). Although Üssing chamber analyses have suggested that both of these processes may correlate with anti-CD3-induced diarrhea, the role of anion secretion in this diarrhea is controversial; one study attributed the diarrhea to increased active anion secretion (30), while another showed that cystic fibrosis transmembrane conductance regulator-mediated (CFTR-mediated) $\mathrm{Cl}^{-}$secretion was suppressed by anti-CD3 treatment (14). To determine whether CFTR-mediated $\mathrm{Cl}^{-}$secretion was required for the net water secretion and barrier defect observed after $\mathrm{T}$ cell activation, we assessed anti-CD3-induced diarrhea in CFTR $\triangle \mathrm{F} 508$ mice, which harbor the most common human CFTR mutation (36). Anti-CD3 treatment of CFTR $\triangle F 508$ mice induced net water secretion (Figure 3D, third bar) and increased BSA paracellular flux (Figure 3E, third bar) that were similar to those seen in wild-type animals treated with anti-CD3. To inhibit $\mathrm{Ca}^{2+}$-activated chloride channels, mice were treated with niflumic acid. This did not inhibit net water secretion (Figure 3D, fourth bar) or increases in BSA flux (Figure 3E, fourth bar) after anti-CD3 injection. Thus, neither CFTR-nor $\mathrm{Ca}^{2+}$-activated chlo- 
A

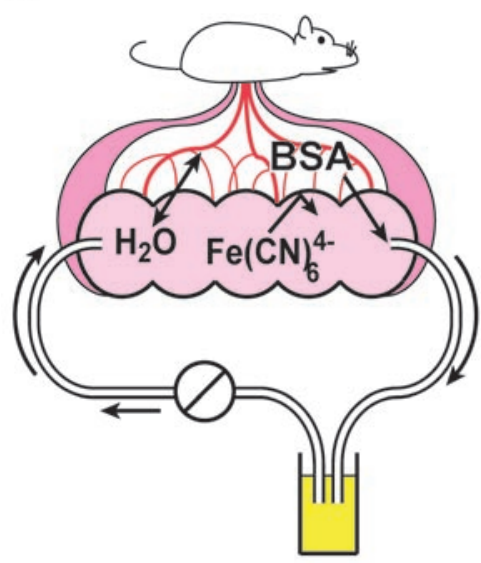

B

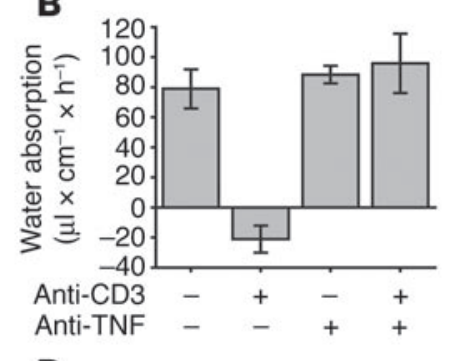

D

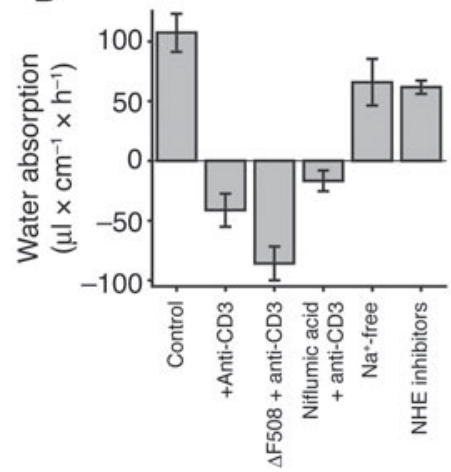

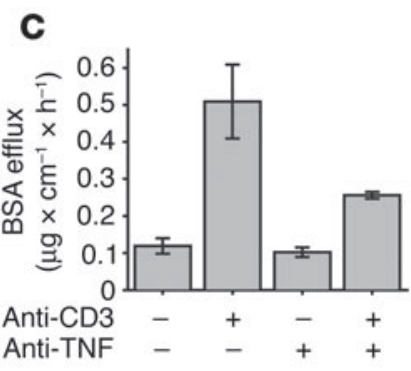

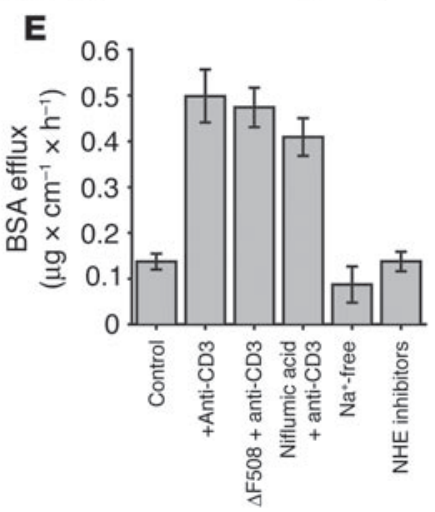

\section{Figure 3}

In vivo measurement of water movement and paracellular flux in anti-CD3-induced diarrhea. (A) An in vivo perfusion system was developed to measure water movement and paracellular flux in a segment of intestine with an intact neurovascular supply. Water movement was determined via changes in the concentration of ferrocyanide in the perfusate, while paracellular flux was measured by the movement of intravenously injected fluorescent-tagged BSA into the perfusate. (B) In control mice, water was absorbed. Consistent with the development of diarrhea, anti-CD3 treatment reversed net water flow, resulting in net secretion. These changes were prevented by injection of a TNF-neutralizing antibody ( $n=4)$. (C) BSA flux into the lumen of a perfused segment of small intestine, a measurement of paracellular macromolecular flux, was increased $340 \% \pm 35 \%$ after antiCD3 injection. Treatment with TNF-neutralizing antibody prevented $59 \% \pm 5 \%$ of this anti-CD3-induced increase in BSA flux $(n=4)$. (D) Net water secretion occurred in anti-CD3-treated CFTR $\Delta$ F508 ( $\Delta$ F508) mice or in wild-type mice perfused with $100 \mu \mathrm{M}$ niflumic acid, indicating that $\mathrm{Cl}^{-}$secretion via CFTR or $\mathrm{Ca}^{2+-}$-activated chloride channels is not necessary for water secretion after $\mathrm{T}$ cell activation. Inhibition of $\mathrm{Na}^{+}$absorption in control animals using either $\mathrm{Na}^{+}$-free perfusate or the NHE inhibitors HOE694 $(200 \mu \mathrm{M})$ and S3226 $(10 \mu \mathrm{M})$ resulted in decreased absorption but did not cause net secretion of water $(n=3)$. (E) Increased paracellular flux also occurred in anti-CD3-treated CFTR $\Delta$ F508 mice or mice treated with niflumic acid but was not induced by perfusion with $\mathrm{Na}^{+}$-free perfusate or the NHE inhibitors HOE694 and S3226 without anti-CD3 treatment $(n=3)$.

ride channel-mediated $\mathrm{Cl}^{-}$secretion are necessary for development of diarrhea or barrier defects following systemic $\mathrm{T}$ cell activation.

We also considered the possibility that the diarrhea observed was due to inhibition of $\mathrm{Na}^{+}$absorption. This would be consistent with previous Üssing chamber analyses demonstrating inhibition of nearly $90 \%$ of NHE2- and NHE3-dependent $\mathrm{Na}^{+}$absorption 3 hours after anti-CD3 treatment. We used 2 different approaches to block NHE2- and NHE3-dependent $\mathrm{Na}^{+}$absorption in the absence anti-CD3 treatment. In the first approach, $\mathrm{Na}^{+}$was replaced by $N$-methyl-D-glucamine in the luminal perfusate. Alternatively, NHE2 and NHE3 were blocked with pharmacological inhibitors S3326 and HOE694. In the standard slightly hypoosmotic perfusion solution used in these studies, both of these approaches reduced net water absorption (Figure 3D, right 2 bars), consistent with induction of $\mathrm{Na}^{+}$malabsorption. However, neither treatment actually reversed net water movement. This may be due to the absorption-promoting nature of the hypoosmotic perfusion solution. Consistent with this, a similar study comparing hypoosmotic and isoosmotic perfusion solutions was performed. This showed that, in the presence of luminal $\mathrm{Na}^{+}$, less water absorption occurred using the isoosmotic perfusion solution. Moreover, replacement of $\mathrm{Na}^{+}$in the isoosmotic perfusion solution completely prevented water absorption and allowed net water movement into the lumen (data not shown). Thus, although $\mathrm{Na}^{+}$malabsorption could disrupt net absorption, $\mathrm{Na}^{+}$malabsorption alone was insufficient to induce massive diarrhea similar to that seen after anti-CD3 injection.

We also asked whether $\mathrm{Na}^{+}$malabsorption alone was able to alter paracellular permeability. Under either hypoosmotic (Figure 3E, right 2 bars) or isoosmotic (data not shown) perfusion conditions, induction of $\mathrm{Na}^{+}$malabsorption was insufficient to cause increased paracellular flux of tagged BSA. Thus, although $\mathrm{Na}^{+}$ malabsorption can impact net water absorption, $\mathrm{Na}^{+}$malabsorption alone is insufficient to cause the diarrhea and barrier defects associated with systemic $T$ cell activation.

T cell activation disrupts tight junction structure. The data presented above show that active $\mathrm{Cl}^{-}$secretion is not necessary and $\mathrm{Na}^{+}$malabsorption is not sufficient for anti-CD3-induced diarrhea to occur. However, the increased passive paracellular flux that occurred also correlated with development of diarrhea. This barrier dysfunction was not due to mucosal ulceration or epithelial apoptosis, but rather occurred in the presence of an intact epithelial cell layer. Thus, we asked whether the intestinal epithelial tight junction, the primary determinant of paracellular flux in intact epithelia, was disrupted following systemic $T$ cell activation. We first examined the distribution of several members of the claudin family of transmembrane junctional proteins (Figure 4A). We found that, in mouse jejunal vil- 

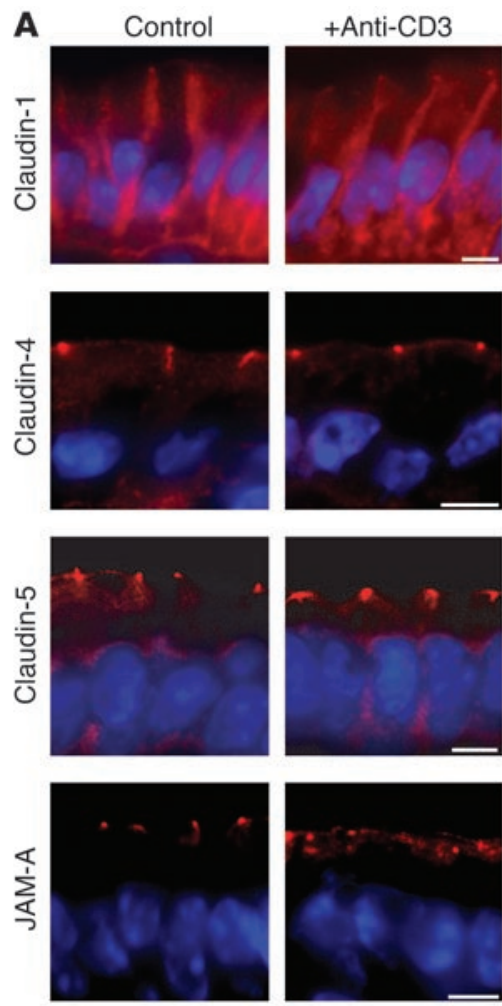
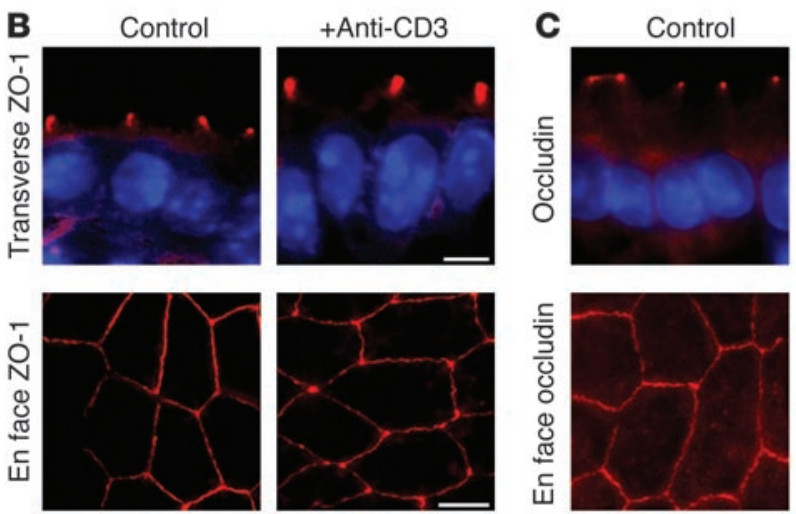

D

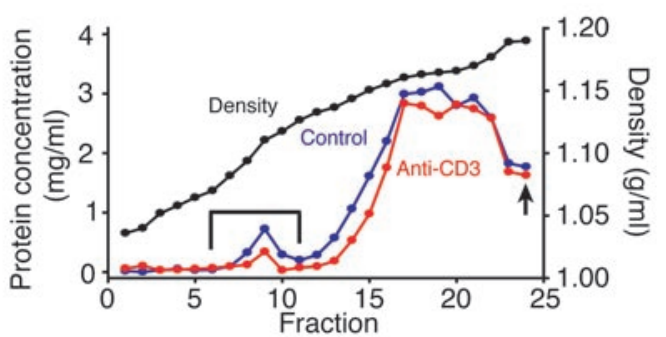

\section{E}

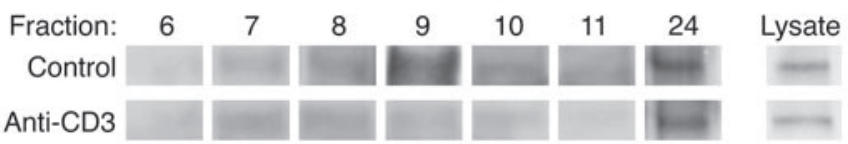

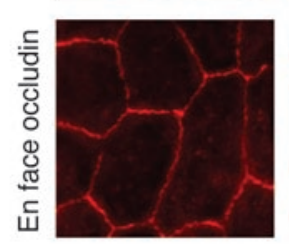

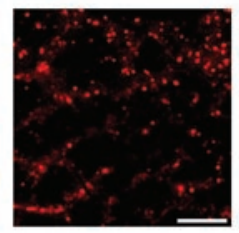

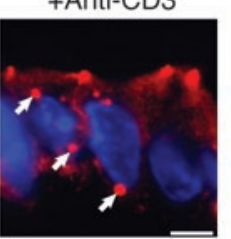

Lysate

\section{Figure 4}

Changes in tight junction morphology after anti-CD3 treatment. (A) Immunofluorescent localization of the tight junction proteins claudin-1, claudin-4, claudin-5, and JAM-A in the small intestinal epithelium of wild-type mice demonstrates the localization of these proteins to the tight junction before (left panels) and 3 hours after (right panels) anti-CD3 treatment. Claudin-1, claudin-4, and claudin-5 distributions were not affected by antiCD3 treatment. However, JAM-A demonstrated limited redistribution to intracellular sites after anti-CD3 treatment. (B) ZO-1 distribution appeared unchanged in transverse sections, but when viewed in en face sections, ZO-1 adopted a thinner, more sinuous distribution in anti-CD3-treated tissue. (C) Occludin was localized to the tight junction in control tissue, but after anti-CD3 treatment, occludin was internalized into intracellular vesicles (arrows), visible in both transverse and en face sections. Scale bars in A-C, $5 \mu \mathrm{m}$. (D) Detergent-insoluble subcellular fractions of jejunal epithelial cells were isolated on isopycnic sucrose gradients (black line). Protein concentration was determined for each fraction of gradients prepared from control (blue line) and anti-CD3-treated (red line) mice. A protein peak associated with the low-density, detergent-insoluble membrane fraction was present (bracket). The arrow designates the insoluble high-density pellet (fraction 24). These fractions were assessed by immunoblot. (E) Immunoblots for occludin in lysate (diluted), pellet, and low-density, detergent-insoluble membrane fractions showed loss of occludin from the detergent-insoluble membrane fraction after anti-CD3 treatment.

lous enterocytes, claudin-1 was localized both to the tight junction and along the lateral membranes. This distribution was not altered by anti-CD3 treatment. Claudin-4 and claudin-5 were specifically localized to the epithelial tight junction. As with claudin-1, there was no detectable change in claudin- 4 or -5 distribution following anti-CD3 treatment (Figure 4A). Similar to previous studies (37), we found claudin-2 within tight junctions of crypt, but not villous, enterocytes. The distribution of claudin-2 in crypt epithelium was not affected by anti-CD3 treatment (data not shown).

Examination of the tight junction protein junctional adhesion molecule A (JAM-A) showed that it is localized to the tight junction in control tissues (Figure 4A). However, displacement from the tight junction and internalization were seen in a minority of cells following anti-CD3 treatment. This JAM-A redistribution occurred predominantly within villous epithelium and was observed in $12 \% \pm 2 \%$ of epithelial cells in the upper half of villi after anti-CD3 treatment, compared with $1 \% \pm 1 \%$ in control tissue $(P=0.002)$.

Zonula occludens-1 (ZO-1), a cytoplasmic plaque tight junction protein, was only detected at the tight junction of jejunal vil- lous enterocytes in both control and anti-CD3-treated animals, and its appearance was similar when cells were viewed in crosssection (Figure 4B). However, when viewed en face, $\mathrm{ZO}-1 \mathrm{dem}-$ onstrated an altered appearance after anti-CD3 treatment. In untreated tissue, $\mathrm{ZO}-1$ was found in a bright line at the tight junction, forming the classic "chicken wire" appearance (Figure 4B). However, after anti-CD3 treatment, $\mathrm{ZO}-1$ staining became thinner and more sinuous (Figure 4B). No intracellular ZO-1 deposits were detected after anti-CD3 treatment.

The distribution of the transmembrane tight junction protein occludin was grossly altered following anti-CD3 treatment. In control jejunal villous enterocytes, occludin was exclusively localized to the tight junction. This was dramatically rearranged to include intracellular occludin deposits as well as junction-associated occludin after $T$ cell activation (Figure $4 \mathrm{C}$ ). This occludin redistribution was even more striking when viewed en face (Figure 4C), where the classic pattern of tight junction staining was nearly completely lost after anti-CD3 treatment. Occludin internalization was most widespread in the villous tips, where it was detectable in $39 \% \pm 7 \%$ 


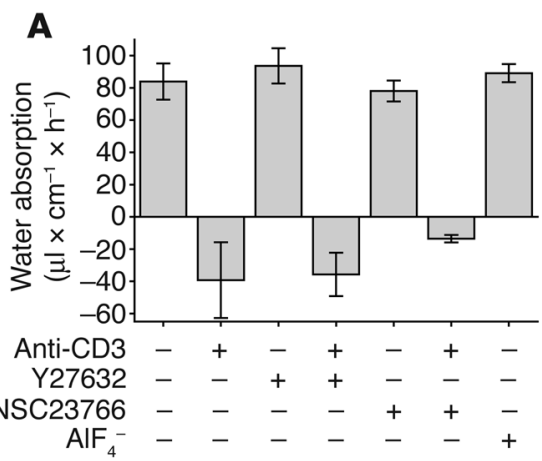

B

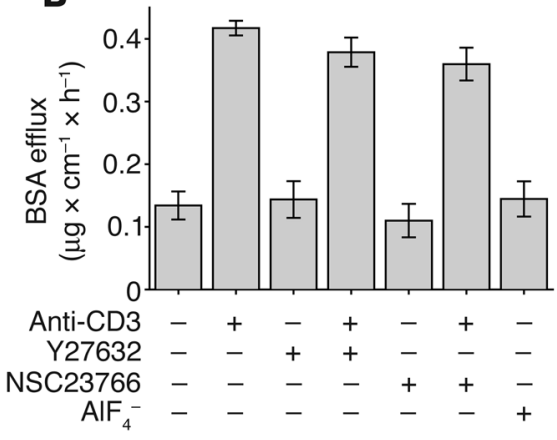

of enterocytes in the upper half of the villi after anti-CD3 treatment but only in $2 \% \pm 2 \%$ of villous enterocytes in control tissue $(P=0.001)$. This appearance is consistent with vesicular endocytosis of occludin, which has been reported in cultured monolayers after TNF- $\alpha$ exposure $(15,20)$ but has not previously been observed in tissues responding to in vivo stimuli.

To examine this profound occludin redistribution biochemically, we isolated low-density, detergent-insoluble glycolipid- and cholesterol-rich membrane fractions. In cultured epithelia, these fractions have been shown to be enriched in tight junction-derived membranes (38). Subcellular fractions were separated on isopycnic density gradients using jejunal epithelia isolated from control and anti-CD3treated mice (Figure 4D). Immunoblot of the low-density, detergentinsoluble membrane fractions revealed a marked loss of occludin in preparations from anti-CD3-treated mice relative to control mice (Figure 4E). In contrast, total cellular occludin as well as occludin recovery in the detergent-insoluble pellet were unchanged. Thus, anti-CD3 treatment caused biochemical redistribution of occludin out of the detergent-insoluble glycolipid- and cholesterol-rich membrane fraction. This correlates with the occludin internalization observed morphologically. Together, the immunofluorescence microscopy and subcellular fractionation suggest that an ordered rearrangement of tight junction architecture is induced following $\mathrm{T}$ cell activation. This was characterized by occludin and, to a lesser extent, JAM-A internalization and ZO-1 redistribution, while the distribution of claudin proteins remained intact.

GTP-binding proteins are not involved in the development of anti-CD3induced diarrhea. To identify the mechanism by which anti-CD3 treatment leads to intestinal barrier dysfunction and diarrhea, we first investigated the potential role of Rho family GTPases in the development of anti-CD3-induced diarrhea. These signaling molecules are attractive candidates for this process, given that TNF has been reported to activate Rho, Rac, and Cdc42 (39) and that these proteins may regulate tight junction protein distribution and

\section{Figure 5}

Small GTP-binding proteins are not involved in the development of antiCD3-induced water secretion or increased paracellular flux. (A) Net water movement was measured in wild-type mice injected with $200 \mu \mathrm{g}$ of anti-CD3 and perfused with $10 \mu \mathrm{M}$ Y27632, $100 \mu \mathrm{M}$ NSC23766, or $20 \mu \mathrm{M} \mathrm{AlF}_{4}{ }^{-}$as indicated. Neither the Rho kinase inhibitor Y27632 nor the Rac1 inhibitor NSC23766 was able to prevent net water secretion after anti-CD3 treatment, and perfusion with the GTPase activator $\mathrm{AlF}_{4}{ }^{-}$did not induce net water secretion $(n=3)$. (B) Similarly, measurement of paracellular flux in the same treatment groups shown in $\mathbf{A}$ demonstrated that neither Y27632 nor NSC23766 was able to prevent anti-CD3-induced increases in paracellular flux, while $\mathrm{AlF}_{4}^{-}$perfusion did not stimulate increased flux $(n=3)$.

epithelial barrier function (40-43). To investigate the role of Rho family GTPases, we included the Rho kinase inhibitor Y27632 (44) or a Rac1 inhibitor, NSC23766 (45), in the perfusion solution of control and anti-CD3-treated animals. Neither inhibitor caused apparent toxicity, evident by retained water absorption and barrier function in animals treated with drug without anti-CD3 (Figure 5, $\mathrm{A}$ and $\mathrm{B}$ ) and the absence of morphological evidence of toxicity (data not shown). Moreover, neither Rho kinase nor Rac1 inhibition prevented anti-CD3-induced diarrhea or BSA efflux (Figure 5, $A$ and $B$ ). These data suggest that neither Rho kinase nor Rac1 activation are necessary for diarrhea and barrier defects that follow anti-CD3 injection. To evaluate a potential role for other GTP-binding regulators of actin structure, $\mathrm{AlF}_{4}{ }^{-}$, which triggers formation of lamellipodia and membrane ruffles and protrusions $(46,47)$ and can affect barrier function (42), was included in the perfusion solution. This did not result in net water secretion or increased paracellular BSA flux (Figure 5, A and B) without anti-CD3 treatment. Therefore, these results demonstrate that Rho kinase, Rac1, and other GTP-binding regulators of actin structure are not involved in the diarrhea that occurs following systemic T cell activation.

$T$ cell activation induces phosphorylation of epithelial MLC. To more closely examine the changes in tight junction morphology caused by anti-CD3 treatment, we evaluated the ultrastructure of jejunal villous enterocytes in control and anti-CD3-treated mice. Enterocytes from control mice demonstrated the characteristic kiss of adjacent membranes that defines the tight junction (Figure 6A). In contrast, this ordered appearance was disrupted in enterocytes from anti-CD3-treated mice. Moreover, the cytoplasmic density surrounding the tight junction was increased in a manner consistent with cytoskeletal condensation (Figure 6A, arrow). Thus, the alterations in barrier function induced by $\mathrm{T}$ cell activation are accompanied by changes in the ultrastructural morphology of the tight junction and the perijunctional cytoskeleton.

The actomyosin condensation around the tight junction observed after $T$ cell activation closely resembles the changes in epithelial tight junction structure following initiation of $\mathrm{Na}^{+}$-glucose cotransport (48), a process that is dependent on phosphorylation of MLC within the perijunctional actomyosin ring $(22,49)$. Thus, we asked whether $T$ cell activation was associated with increased epithelial MLC phosphorylation. Immunofluorescence detection of phosphorylated MLC 3 hours after anti-CD3 injection revealed a marked increase in MLC phosphorylation within the perijunctional actomyosin ring of jejunal villous enterocytes (Figure 6B). To further assess increases in MLC phosphorylation, we measured MLC phosphorylation in isolated jejunal epithelial cells. MLC phosphorylation was significantly increased 3 hours 
A

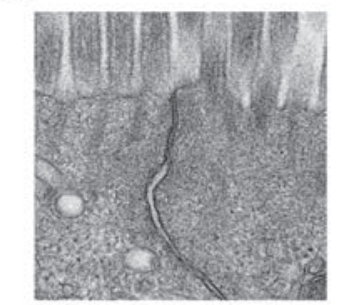

B

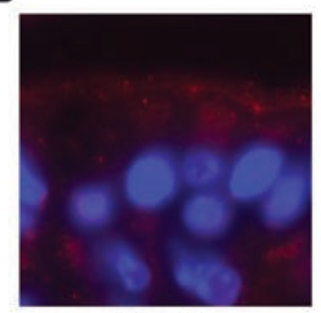

Control

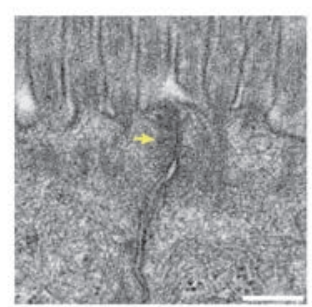

+ Anti-CD3

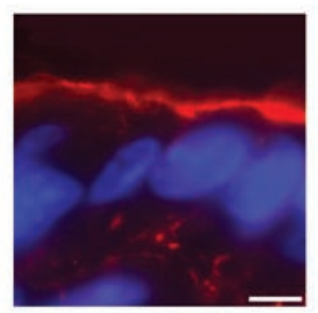

+ Anti-CD3
C

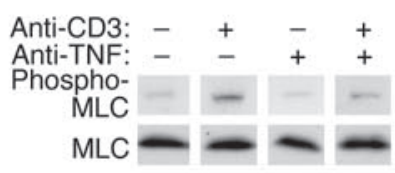

D

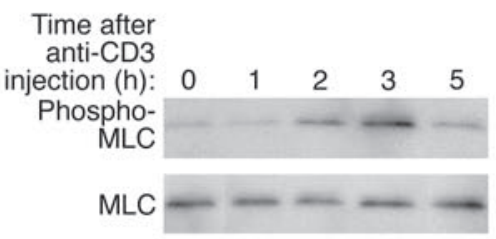

Time after anti-CD3

injection (h): $\begin{array}{lllll}0 & 1 & 2 & 3 & 5\end{array}$

MLC

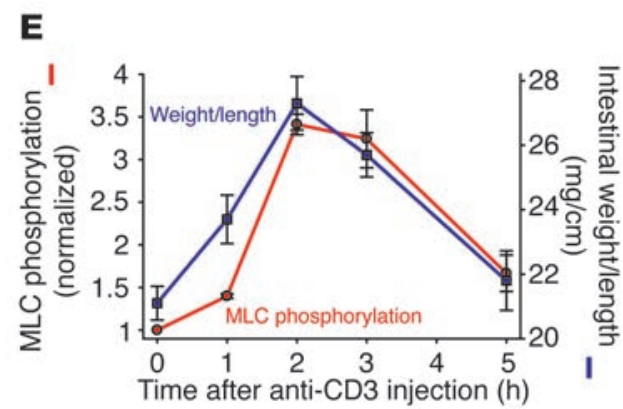

Figure 6

Systemic T cell activation increases intestinal epithelial MLC phosphorylation. (A) Electron micrographs of villous enterocytes from jejunum of control and anti-CD3-treated mice demonstrate disruption of the tight junction and marked perijunctional cytoskeletal condensation (arrow) after T cell activation (scale bar, $250 \mathrm{~nm}$ ). (B) Phosphorylated MLC (red) in control intestinal villi was localized to the perijunctional actomyosin ring and demonstrates enhancement at cell junctions. Three hours after anti-CD3 treatment, MLC phosphorylation was markedly increased. Matched exposures are shown (scale bar, $5 \mu \mathrm{m}$ ). (C) Intestinal epithelial MLC phosphorylation, determined by immunoblot for phosphorylated and total MLC in isolated intestinal epithelial cells, revealed an increase in MLC phosphorylation 3 hours after anti-CD3 injection. This increase in MLC phosphorylation was largely prevented by the TNF-neutralizing antibody. (D) Immunoblots of phosphorylated MLC at several time points after anti-CD3 injection showed that epithelial MLC phosphorylation peaked 2-3 hours after anti-CD3 injection and returned to baseline levels within 5 hours after anti-CD3 injection. (E) Quantitative analysis of the blots showed that MLC phosphorylation (red line) increased and decreased in parallel with changes in intestinal weight-to-length ratio (blue line) induced by T cell activation $(n=3)$.

after anti-CD3 injection (Figure 6C). This did not occur in mice injected with anti-CD3 and the TNF-neutralizing antibody, indicating that TNF-mediated signaling drives MLC phosphorylation after anti-CD3 treatment (Figure 6C).

To determine the kinetics of epithelial MLC phosphorylation after anti-CD3 treatment, we measured phosphorylation at intervals after anti-CD3 injection (Figure 6D). Quantitative analysis showed that MLC phosphorylation increased more than 3-fold at 2 and 3 hours after anti-CD3 injection (Figure 6E). MLC phosphorylation then fell to levels similar to those for control epithelia within 5 hours after anti-CD3 injection (Figure 6E). This rise and fall in MLC phosphorylation correlated with the development and resolution of diarrhea measured as intestinal weight-to-length ratio, suggesting that epithelial MLC phosphorylation is closely linked to the development of overt symptoms of disease.

Mice lacking the 210-kDa MLCK are protected from T cell activationinduced diarrhea. Total MLC phosphorylation is regulated by the balance of several kinases and phosphatases $(8,50-53)$. In intestinal epithelia the primary kinase that phosphorylates MLC is the $210-\mathrm{kDa}$ long MLCK isoform $(24,54,55)$. Thus, to evaluate the role of epithelial MLCK in diarrhea induced by anti-CD3 injection, we studied knockout mice lacking the 210-kDa MLCK (56). To first determine whether this MLCK knockout attenuated local immune responses generated by anti-CD3 injection, we examined cytokine induction in these mice. Levels of mucosal IFN- $\gamma$ (Figure 7A) and TNF- $\alpha$ (Figure 7B) mRNA transcripts were significantly increased after anti-CD3 injection in both wild-type and long
MLCK-knockout animals. Consistent with this, examination of the serosa after anti-CD3 treatment of long MLCK-knockout mice demonstrated vascular injection and erythema similar to those in wild-type mice. Thus, the $210-\mathrm{kDa}$ MLCK is not required for cytokine induction in response to anti-CD3.

Basal jejunal epithelial MLC phosphorylation was similar in wild-type and long MLCK-knockout animals (Figure 7C), suggesting that this kinase is not the only regulator of MLC phosphorylation in intestinal epithelia. Consistent with this, intestinal histology, including villous height and ratio of villus to total villus-crypt length were not altered in MLCK-knockout mice. However, jejunal epithelial MLC phosphorylation did not increase after anti-CD3 injection in MLCK-knockout mice (Figure 7C). Thus, although the $210-\mathrm{kDa}$ MLCK is not essential in the absence of stress, this MLCK isoform is required for increased epithelial MLC phosphorylation following systemic $\mathrm{T}$ cell activation.

Given that an immune response is generated in long MLCKknockout mice without increases in epithelial MLC phosphorylation, these mice represent an ideal model for determining which aspects of anti-CD3-induced disease require epithelial MLC phosphorylation. Although MLCK-knockout animals treated with antiCD3 did exhibit outward evidence of immune activation after antiCD3 treatment, including mild piloerection and lethargy similar to those in wild-type mice, there was no external evidence of diarrhea, and little fluid accumulation was observed in the intestinal lumen. This was confirmed by analysis of small intestinal weight-to-length ratio 3 hours after anti-CD3 injection. In contrast to wild-type mice, 


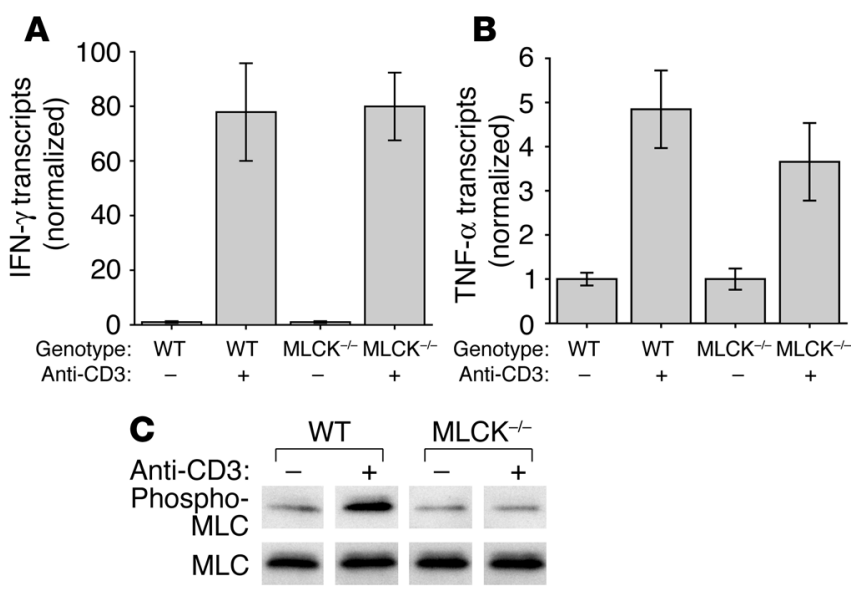

anti-CD3 injection did not cause a significant increase in weight-tolength ratio in MLCK-knockout mice (Figure 8A).

To assess water movement and paracellular macromolecular flux quantitatively in MLCK-knockout mice, we utilized the in vivo perfusion system described above. In the absence of anti-CD3 treatment, net water absorption and paracellular BSA flux were similar in wild-type and MLCK-knockout mice (Figure 8, B and C). However, in contrast to wild-type mice, anti-CD3 injection did not reverse the direction of water movement to net secretion in MLCK-knockout mice (Figure 8B). Moreover, paracellular flux was not increased after anti-CD3 treatment in MLCK-knockout mice (Figure 8C). Thus, the long MLCK that is expressed in intestinal epithelia and is necessary for MLC phosphorylation is also required for development of diarrhea and barrier dysfunction following systemic $T$ cell activation.

To determine whether long MLCK knockout also prevented reorganization of the tight junction after anti-CD3 treatment, we assessed the localization of occludin, whose redistribution to intracellular structures was the most prominent change induced by anti-CD3 treatment of wild-type mice. In contrast to wild-type mice, occludin remained confined to the tight junction following anti-CD3 treatment of long MLCK-knockout mice (Figure 8D). Only $4 \% \pm 1 \%$ of villous enterocytes in long MLCK-knockout animals contained internalized occludin, significantly less than the $39 \% \pm 7 \%$ seen in control animals $(P=0.001)$. Thus, long MLCK is necessary for both functional tight junction disruption (i.e., increased paracellular flux) and morphological tight junction disruption following systemic $\mathrm{T}$ cell activation. This suggests that these changes contribute directly to the diarrhea that occurs in wild-type, but not long MLCK-knockout, mice.

Treatment with a membrane-permeant inhibitor of MLCK prevents $T$ cell activation-induced diarrhea. The striking protection of long MLCK-knockout mice from anti-CD3-induced disease suggests that intestinal epithelial MLCK may be a novel target for nonimmunosuppressive therapy of immune-mediated diarrhea. We have recently described a membrane-permeant inhibitor of MLCK (PIK) (11). This short peptide mimics the regulatory domain of MLCK, which confers specificity, and also has sequence similarity to protein transduction domains, e.g., of HIV-1 Tat, that allow cytoplasmic access (57). When used in vitro, PIK reversed cytokineinduced reductions in transepithelial electrical resistance, an indicator of barrier function, in cultured epithelial monolayers (11). However, the originally described PIK is unsuitable for use in vivo

\section{Figure 7}

Mice lacking the 210-kDa MLCK demonstrate cytokine induction after anti-CD3 treatment but do not increase epithelial MLC phosphorylation. (A) Mucosal IFN- $\gamma$ transcription was markedly increased 3 hours after anti-CD3 injection in both wild-type and $\mathrm{MLCK}^{--}$mice $(n=3)$. (B) Mucosal TNF- $\alpha$ transcription was markedly increased 3 hours after anti-CD3 injection in wild-type and $\mathrm{MLCK}^{-1-}$ mice $(n=3)$. (C) Western blot analysis of MLC phosphorylation in isolated epithelial cells of wildtype and $\mathrm{MLCK}^{-/-}$mice 3 hours after anti-CD3 treatment showed a large increase in MLC phosphorylation in wild-type mice that did not occur in $\mathrm{MLCK}^{-/}$mice.

due to its susceptibility to proteolysis in the intestinal lumen (58). To overcome this limitation, we developed a stable PIK analog that possessed the same membrane permeance and specificity for MLCK as the parent compound but was approximately 4,000-fold more resistant to proteolysis (58). Because our previous in vitro work applied PIK only to the apical surface of cultured monolayers, stable PIK was added directly to the perfusion solution in our in vivo permeability assay, a route of administration analogous to oral delivery. To determine the distribution of stable PIK delivered in this manner, the jejunal segment was perfused with biotinylated PIK, which was then detected with fluorochrome-conjugated streptavidin. Stable PIK was detected in intestinal epithelia throughout the villus and crypt (Figure 9A). However, PIK was not detectable within lamina propria stromal cells or underlying muscularis. This agrees with previous reports (59) that protein transduction domains are not suitable for systemic delivery across epithelial-lined surfaces. In this case, that property is an advantage, as it suggests failure of systemic delivery and therefore implies that systemic toxicity should not limit therapeutic use of stable PIK. Consistent with this, no systemic effects were noted at perfused stable PIK concentrations as high as $250 \mu \mathrm{M}$. Gross and histological examination of intestinal segments perfused with $250 \mu \mathrm{M}$ stable PIK for 2 hours also failed to show any evidence of toxicity (Figure 9B). Moreover, the selective delivery of stable PIK to the epithelium did not affect the mucosal cytokine response to anti-CD3, as induction of mucosal TNF- $\alpha$ and IFN- $\gamma$ transcription after anti-CD3 injection were similar in PIK-treated and untreated jejunum (Figure 9, C and D). Thus, stable PIK delivered luminally accesses crypt and villous epithelia but not subepithelial compartments and does not cause systemic toxicity.

To determine whether stable PIK is able to prevent epithelial MLC phosphorylation after anti-CD3 treatment, epithelia were isolated from perfused jejunal segments. Stable PIK completely prevented increased phosphorylation after anti-CD3 injection (Figure 9E). Thus, stable PIK applied in the perfusion solution is targeted to the appropriate tissue compartment, the epithelium, and accomplishes its desired biochemical action, prevention of MLC phosphorylation.

To determine whether this inhibition of MLCK-mediated MLC phosphorylation also prevented redistribution of tight junction proteins, occludin localization was assessed in jejunal segments perfused with $80 \mu \mathrm{M}$ stable PIK. Treatment with PIK significantly reduced occludin internalization after anti-CD3 treatment; only $5 \% \pm 2 \%$ of villous enterocytes in PIK-treated mice contained internalized occludin, compared with $39 \% \pm 7 \%$ of enterocytes in anti-CD3-treated mice without PIK $(P=0.002$; Figure 10A). Thus, MLCK inhibition with stable PIK prevents anti-CD3-mediated tight junction reorganization. 

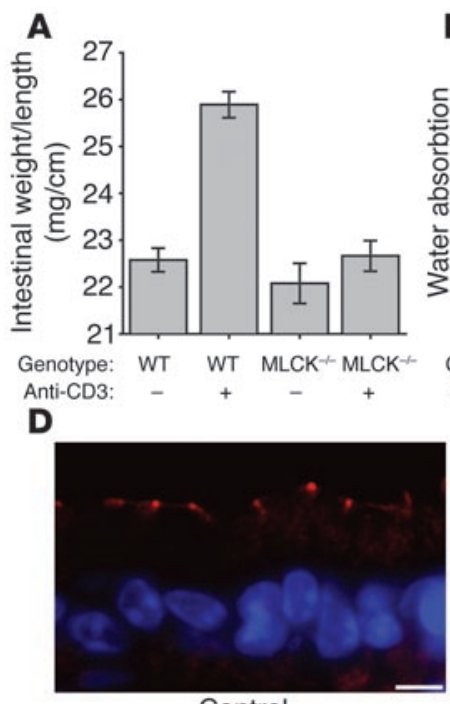

Control
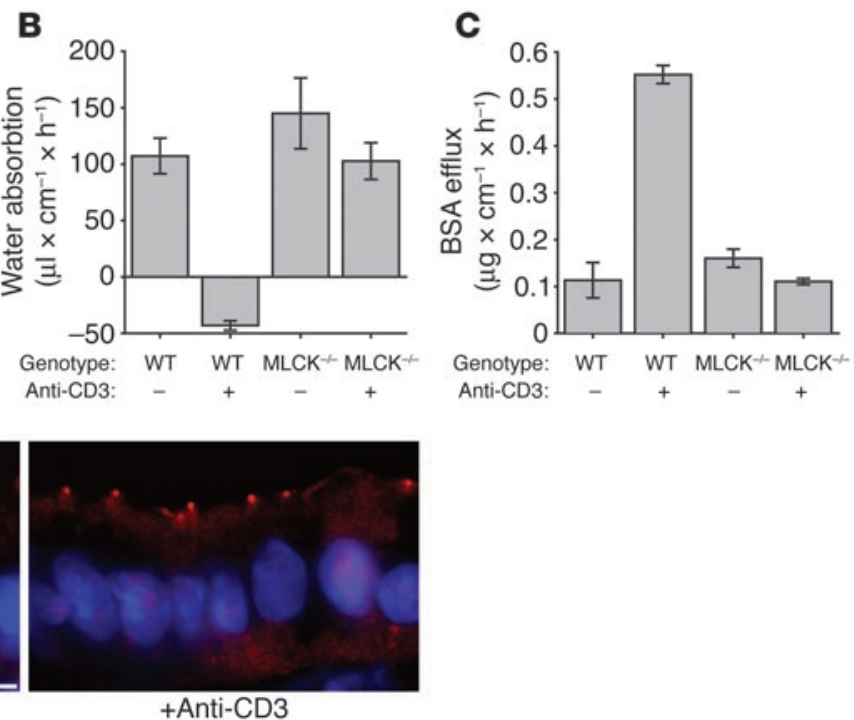

Figure 8

210-kDa $\mathrm{MLCK}^{-1-}$ mice are protected from anti-CD3-induced diarrhea. (A) Intestinal weight-to-length ratio did not increase in $\mathrm{MLCK}^{-1-}$ mice 3 hours after anti-CD3 treatment, while wild-type mice showed a large increase in weight-to-length ratio $(P=0.52 ; n=4)(B)$ Consistent with the lack of an increase in weightto-length ratio, perfused segments of small intestine in $\mathrm{MLCK}^{-1-}$ mice treated with anti-CD3 did not show significant changes in water movement relative to untreated $\mathrm{MLCK}^{-1-}$ mice $(P=0.35 ; n=4)$. (C) Paracellular permeability, indicated by BSA efflux, did not increase in $\mathrm{MLCK}^{-1-}$ mice treated with anti-CD3 relative to untreated $\mathrm{MLCK}^{-1-}$ mice $(P=0.12 ; n=4)$. (D) Immunofluorescent detection of occludin in intestinal epithelia of $\mathrm{MLCK}^{-/-}$mice demonstrated that occludin remained confined to the tight junction 3 hours after anti-CD3 treatment, without any evidence of internalization (scale bar, $5 \mu \mathrm{m}$ ).

The results with the long MLCK-knockout mouse suggest that epithelial MLC phosphorylation is critical for the diarrhea induced by anti-CD3 treatment. However, long MLCK is also expressed in nonepithelial cell types $(54,60,61)$. Thus, it is possible that the protective effect of long MLCK knockout was due to loss of MLCK function in nonepithelial cells. PIK overcomes this limitation, since the similarity of stable PIK to the pseudosubstrate domain of MLCK confers extremely high target specificity, and the protein transduction domain present in PIK results in its restriction to the epithelium after luminal application. Thus, stable PIK represents a pharmacological approach to specifically inhibit only intestinal epithelial MLCK. Stable PIK prevented anti-CD3-induced increases in paracellular permeability, as assessed by BSA flux (Figure 10B). This inhibition was dose dependent over a concentration range consistent with the $\mathrm{IC}_{50}$ of stable PIK (58). At the highest dose tested, $250 \mu \mathrm{M}$, stable PIK reduced paracellular permeability to the level observed in control animals that were not treated with stable PIK or antiCD3. Thus, specific inhibition of intestinal epithelial MLCK prevents barrier dysfunction induced by $\mathrm{T}$ cell activation.

Stable PIK did not affect net water absorption in mice not treated with anti-CD3 (Figure 10C). In anti-CD3-treated mice, stable PIK restored net water absorption in a dose-dependent manner that paralleled the effect on paracellular permeability (Figure 10C). However, even at the maximal dose of stable PIK tested, water absorption in anti-CD3-treated mice was less than that in control mice $(P=0.03$; Figure 9C). This observation suggests that while MLCK-dependent MLC phosphorylation is necessary for diarrhea to occur, it is not the only mechanism of reduced absorption following $\mathrm{T}$ cell activation. Despite this, the data clearly show that inhibition of MLCK with stable PIK completely prevents diarrhea and epithelial barrier dysfunction in this in vivo model of acute immune-driven intestinal disease.

\section{Discussion}

Increased intestinal paracellular permeability often accompanies diarrhea in gastrointestinal diseases, including inflammatory bowel disease, graft versus host disease, and infectious enterocolitis (1-6). However, the role of permeability defects, or barrier dysfunction, in disease pathogenesis in vivo remains poorly defined. This is partly due to the absence of specific agents that can restore the epithelial barrier without suppression of the overall inflammatory process. Our aims in this study were to define the mechanisms of epithelial barrier dysfunction in immune-mediated diarrheal disease, to specifically reverse that barrier dysfunction without limiting immune activation, and therefore to determine the role of barrier dysfunction in disease pathogenesis. We studied a mouse model of acute immunemediated diarrhea that is similar to the TNF-dependent diarrhea seen in patients treated with anti-CD3 monoclonal antibody OKT3 $(25,26)$. The data show that in vivo epithelial barrier dysfunction, defined as both increased paracellular protein flux and tight junction disruption, is mediated by MLCK-dependent MLC phosphorylation. Both genetic and pharmacological MLCK inhibition prevented in vivo epithelial barrier dysfunction following systemic $\mathrm{T}$ cell activation. Moreover, these same interventions prevented the diarrhea that occurred in wild-type mice. These studies therefore provide unequivocal evidence that MLCK regulates intestinal epithelial paracellular permeability in vivo and are the first to our knowledge to show the central role of this regulation in diarrhea disease.

Anti-CD3-induced T cell activation is a well-established model of acute immune-mediated intestinal disease involving epithelial barrier dysfunction and diarrhea. Previous reports have suggested both epithelial apoptosis (62) and active ion secretion (30) as important events in the development of these in vivo symptoms. In vitro studies also suggest that TNF can induce apoptosis of model intestinal epithelia. For example, conductance scanning studies suggest that apoptosis is responsible for approximately half of the TNF-mediated barrier defect induced in HT-29 monolayers, with increases in tight junction permeability accounting for the remainder (19). However, in agreement with a previous study that reported apoptosis beginning 8-12 hours after anti-CD3 injection (62), we found no histological or biochemical evidence of epithelial apoptosis 3 hours after anti-CD3 treatment, during the peak of anti-CD3-induced diarrhea. Thus, while TNF-mediated epithelial apoptosis may be a long-term consequence of systemic $\mathrm{T}$ cell activation, it does not contribute to the acute diarrhea observed in the first hours after anti-CD3 injection. 

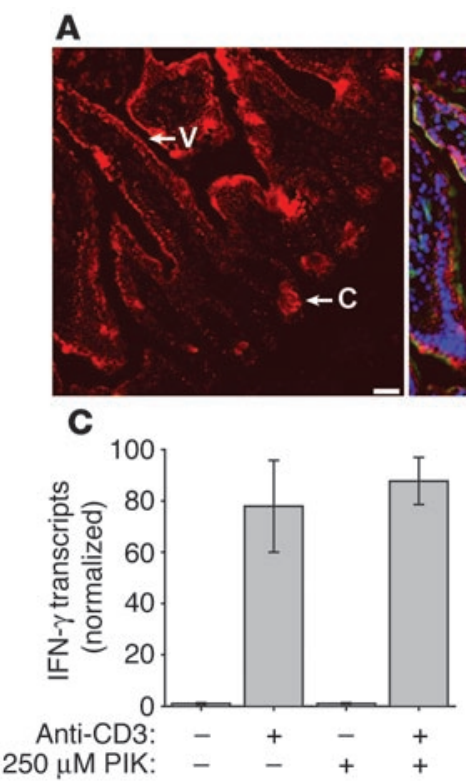

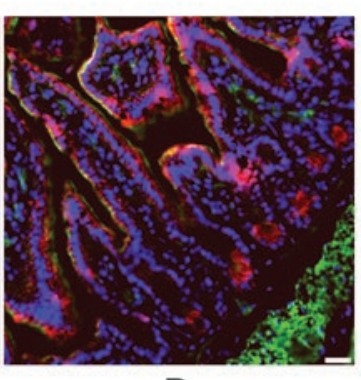

D
B +Anti-CD3

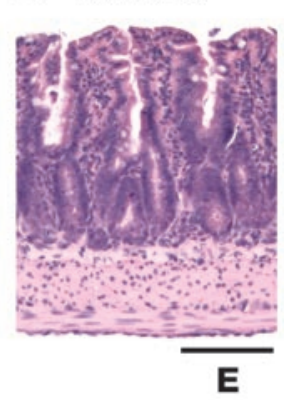

+Anti-CD3 + PIK

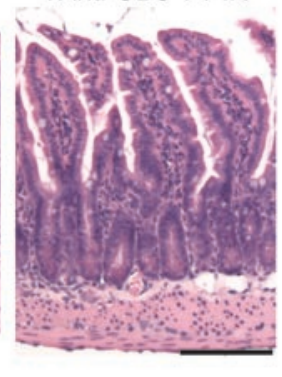

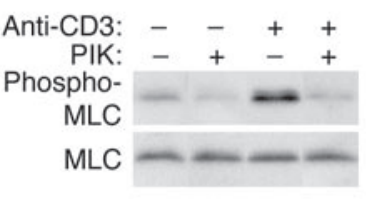

Anti-CD3: 250 मM PIK:

\section{Figure 9}

Treatment of intestinal epithelium with the MLCK inhibitor PIK. (A) The distribution of biotinylated stable PIK after perfusion of a segment of small intestine was detected using fluorescently tagged streptavidin (red). F-actin (green) and DNA (blue) are superimposed in the right panel for orientation. Perfused PIK was present in villous (V) and crypt (C) epithelia but was not seen within the lamina propria, muscularis mucosa, or submucosa (scale bars, $25 \mu \mathrm{m}$ ). (B) H\&E-stained section of anti-CD3-treated intestine with and without PIK treatment showed no evidence of toxicity related to PIK treatment (scale bars, $100 \mu \mathrm{m}$ ). (C) Mucosal IFN- $\gamma$ transcription was markedly increased 3 hours after anti-CD3 injection in both PIK-treated and untreated mice $(n=3)$. (D) Mucosal TNF- $\alpha$ transcription was markedly increased 3 hours after anti-CD3 injection in both PIK-treated and untreated mice $(n=3)$. (E) Western blot analysis of epithelial MLC phosphorylation revealed that the addition of $250 \mu \mathrm{M}$ PIK to the luminal perfusate reduced baseline epithelial MLC phosphorylation and also prevented the large increase in MLC phosphorylation induced 3 hours after anti-CD3 injection.

To assess the contribution of secretory pathways to the development of anti-CD3-induced diarrhea, we attempted to prevent diarrhea by blocking either CFTR- or $\mathrm{Ca}^{2+}$-activated $\mathrm{Cl}^{-}$channel-mediated $\mathrm{Cl}^{-}$secretion. Neither mice carrying a mutant CFTR nor those perfused luminally with niflumic acid, a $\mathrm{Ca}^{2+}$-activated $\mathrm{Cl}^{-}$channel inhibitor, were protected from anti-CD3-induced diarrhea, indicating that neither mechanism of $\mathrm{Cl}^{-}$secretion is necessary for the barrier dysfunction or diarrhea to occur. Thus, although it remains formally possible that another active ion secretory mechanism may be involved in anti-CD3-induced diarrhea (30), neither CFTR- nor $\mathrm{Ca}^{2+}$-activated $\mathrm{Cl}^{-}$channel-mediated $\mathrm{Cl}^{-}$secretion are required.

Studies of tissue excised from anti-CD3-treated animals have also suggested that diarrhea is mediated by suppression of $\mathrm{Na}^{+}$dependent absorptive pathways (14). To test this in vivo, we inhibited $\mathrm{Na}^{+}$-dependent absorption using pharmacological inhibitors or by removing $\mathrm{Na}^{+}$from the luminal perfusate. Both of these treatments caused malabsorption, evident as decreased net water absorption. However, neither induced net water secretion or barrier dysfunction in hypoosmolar perfusate. Thus, malabsorption alone is insufficient to explain the net water secretion, barrier loss, and massive acute diarrhea induced by systemic $\mathrm{T}$ cell activation.

Given that neither $\mathrm{Cl}^{-}$secretion nor $\mathrm{Na}^{+}$malabsorption were sufficient to explain the massive diarrhea or barrier dysfunction that followed systemic $T$ cell activation, we focused our attention on the mechanisms of barrier dysfunction. We found that the development and resolution of diarrhea were closely correlated with the level of epithelial MLC phosphorylation. We have shown this to be a critical physiological regulator of barrier function in cultured monolayers and isolated human intestine
$(22,49)$. It is now clear from a variety of in vitro studies of cultured monolayers that MLC phosphorylation is also responsible for some portion of the barrier dysfunction induced by bacteria $(1,11)$, parasites $(23)$, and cytokines $(11,20,21)$. However, the role of MLC phosphorylation in intestinal disease has not been previously studied in vivo. Our data demonstrate a striking correlation among epithelial MLC phosphorylation, barrier dysfunction, and diarrhea.

Epithelial MLC phosphorylation was accompanied by ultrastructurally evident tight junction reorganization as well as a striking internalization of the transmembrane tight junction proteins occludin and, to a lesser extent, JAM-A. Both occludin and JAM-A internalization have been reported in cultured epithelial monolayers treated with TNF- $\alpha$ and IFN- $\gamma(15,20)$. Similarly, occludin internalization has been observed following extracellular $\mathrm{Ca}^{2+}$ chelation, where myosin motor function has been implicated in tight junction protein endocytosis (63), and following actin depolymerization, where caveolae-mediated endocytosis is required for barrier dysfunction (64). Furthermore, TNF treatment of HT-29 monolayers has been reported to cause a decrease in tight junction strand number in tandem with barrier dysfunction (65), which is a potential consequence of internalization of transmembrane tight junction proteins such as occludin and JAM-A. Despite this abundant in vitro work, these changes have not been previously observed in vivo. Moreover, as discussed below, MLCK inhibition prevented both occludin internalization and barrier dysfunction, suggesting that this tight junction protein internalization may be an important mechanism of the in vivo epithelial barrier dysfunction observed in chronic intestinal disease $(65,66)$. 


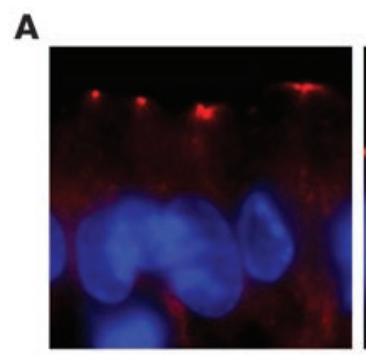

Control

B

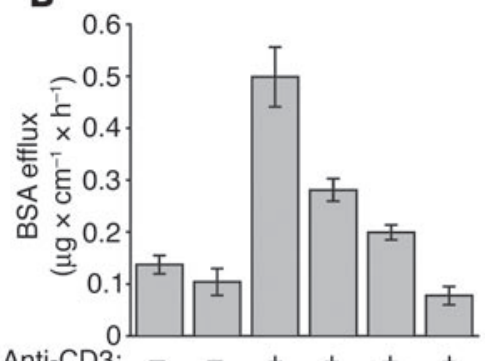

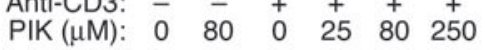
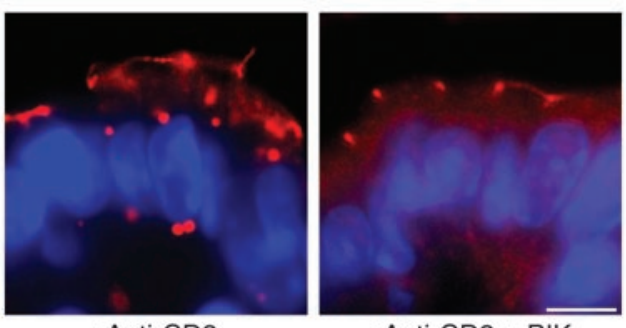

+ Anti-CD3 + PIK

C

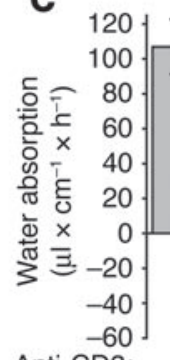

Anti-CD3: PIK $(\mu \mathrm{M})$ :
.

\section{Figure 10}

Treatment with the MLCK inhibitor PIK prevents anti-CD3-mediated tight junction reorganization and diarrhea. (A) Immunofluorescence detection of occludin in the intestinal epithelium of mice treated with anti-CD3 and/or PIK showed that PIK prevents the internalization of occludin associated with anti-CD3 treatment (scale bar, $5 \mu \mathrm{m})$. (B) Increases in paracellular BSA efflux induced by anti-CD3 treatment were reduced by PIK perfusion in a dose-dependent manner $(P=0.015,0 \mu \mathrm{M}$ PIK with antiCD3 vs. $80 \mu \mathrm{M}$ PIK with anti-CD3; $n=4)$. (C) Treatment with $80 \mu \mathrm{M}$ PIK resulted in the restoration of net water absorption in anti-CD3-treated mice $(P=0.006 ; n=4)$.

In addition to preventing structural and functional tight junction disruption, MLCK inhibition prevented diarrhea. Thus, in addition to being the first in vivo analysis of MLC phosphorylation-dependent tight junction regulation in intact intestine, these data are the first to our knowledge to directly demonstrate a role for paracellular barrier dysfunction in diarrheal disease. We used both genetic and pharmacologic approaches to demonstrate this essential role for MLCK. The genetic approach was aided by our recent discovery that only the long isoform of MLCK is expressed in intestinal epithelia (24). Thus, we were able to use mice with a selective knockout of the long isoform (56). Unlike mice in which long and short MLCK isoforms are knocked out (67), these long MLCK-knockout mice are viable and phenotypically normal in the absence of stress. These mice responded to anti-CD3 injection with mucosal immune activation similar to that occurring in wild-type mice but failed to phosphorylate intestinal epithelial MLC. The long MLCK-knockout mice also failed to disrupt tight junction structure or function or to develop diarrhea. Thus, long MLCK expression is required for tight junction disruption and diarrhea following systemic $\mathrm{T}$ cell activation.

The results using long MLCK-knockout mice are the first to our knowledge to show that MLCK is necessary for acute immunemediated diarrhea to occur. However, this isoform is expressed in other tissues. Thus, it is formally possible that loss of long MLCK expression in nonepithelial cell types contributed to the protection from acute immune-mediated diarrhea observed in these mice. We therefore asked whether a specific MLCK inhibitor, PIK, was able to prevent diarrhea. PIK has several unique features when compared with other kinase inhibitors. First, because of the presence of a protein transduction domain (57), luminal PIK can enter but not cross epithelial cells. Thus, PIK only accesses intestinal epithelial cells. This makes inhibition of MLCK in other cell types irrelevant, an advantage in terms of data interpretation as well as the absence of systemic toxicity. Moreover, PIK is highly specific $(11,58,68)$. Thus, PIK overcomes the problems of nonspecificity that have plagued studies using ML-7 and ML-9 (69). The data show that PIK completely prevents both structural and functional tight junction disruption as well as diarrhea following $\mathrm{T}$ cell activation. Therefore, intestinal epithelial MLCK activity is necessary for acute immune-mediated diarrhea to occur.

Our data demonstrate that TNF is the primary cytokine responsible for MLCK activation following anti-CD3 treatment. However, the mechanism by which TNF signaling leads to MLCK activation remains unclear. Based on studies of short MLCK regulation in smooth muscle cells, the primary route for MLCK activation is believed to require $\mathrm{Ca}^{2+}$-dependent calmodulin binding to the MLCK regulatory domain (70). However, TNF- $\alpha$ has not been shown to induce a $\mathrm{Ca}^{2+}$ signal in intestinal epithelia (71). Thus, although further investigation may be warranted, TNF-dependent MLCK activation in the intestinal epithelium may be independent of $\mathrm{Ca}^{2+}$ signaling. We therefore considered alternative mechanisms of MLCK activation. In cultured endothelial cells, cleavage by caspase- 3 can lead to $\mathrm{Ca}^{2+}$-independent long MLCK activation (72). We did not, however, observe caspase- 3 activation or long MLCK cleavage during the development of anti-CD3-induced diarrhea. Thus, caspase-3mediated activation is unlikely to play a role here.

The long MLCK expressed in endothelia and intestinal epithelia (24) differs from short MLCK in that it contains unique amino-terminal domains (73). These include tyrosine residues at positions 464 and 471 that can be phosphorylated by Src family kinases (74). While incompletely characterized, purified $\mathrm{p} 60^{\text {Src }}$ can phosphorylate tyrosine 464 and/or 471 of long MLCK-derived peptides (74), which may also be targets for other kinases. In vitro, long MLCK phosphorylation results in enhanced kinase activity at suboptimal $\mathrm{Ca}^{2+}$ concentrations (74). Thus, stimulation of intestinal epithelial Src family kinases, for example, Brk/Sik (75), by TNF- $\alpha$ could provide $\mathrm{Ca}^{2+}$-independent mechanism of MLCK activation. This model is consistent with in vitro studies showing that TNF- $\alpha$ can activate Src kinase activity via TNF receptor 2 (76) and separate observations that TNF receptor 2 mediates TNF- $\alpha$-induced barrier dysfunction (F. Wang and J.R. Turner, unpublished observations). Thus, it may be interesting to explore the possibility that TNF- $\alpha$-dependent MLCK activation occurs by a novel TNF receptor $2 /$ Src family kinase-dependent mechanism in future studies.

It is notable that, while PIK completely prevents loss of barrier function, net water absorption is only partially restored. Similarly, while long MLCK-knockout mice do not develop barrier dysfunction or diarrhea after anti-CD3 treatment, net water absorption is decreased. Indeed, the net water absorption observed following $\mathrm{T}$ cell activation in both long MLCK-knockout and PIK-treated mice was similar to that which occurred when $\mathrm{Na}^{+}$malabsorption was induced. Thus, while our data clearly show that intestinal epithelial 
MLCK is required for diarrhea to occur, they do not exclude the possibility that malabsorption is also a significant contributor to this diarrhea. For example, it could be that MLCK-mediated barrier dysfunction is required to provide a route for fluid movement into the intestinal lumen and that the osmotic driving force for this water movement is provided by malabsorption. Future identification of agents that selectively disrupt barrier function without altering ion transport processes may allow this hypothesis to be tested.

In summary, the data show that $\mathrm{T}$ cell activation causes intestinal epithelial barrier dysfunction characterized by increased paracellular protein flux, changes in tight junction morphology, and increased MLC phosphorylation. This barrier dysfunction, which is necessary for the development of anti-CD3-mediated diarrhea, is regulated by long MLCK; both genetic knockout and pharmacological MLCK inhibition effectively prevent anti-CD3-induced barrier dysfunction and diarrhea. Therefore, barrier dysfunction is critical to the development of acute immune-mediated diarrheal disease. Inhibition of MLCK may present a novel non-immunomodulatory therapeutic approach to treatment of such disease.

\section{Methods}

Mouse model. Seven- to 10-week-old wild-type C57BL/6, 210-kDa MLCK-/(56), or CFTR $\triangle F 508$ (36) female mice were injected intraperitoneally with $200 \mu \mathrm{g}$ anti-CD3 (clone 2C11) in $200 \mu \mathrm{l}$ of PBS or vehicle alone. Mice were then used for intestinal permeability assays or sacrificed for tissue harvest. Harvested tissues were snap-frozen in OCT for immunofluorescence, placed in TRIzol (Invitrogen Corp.) for mRNA analysis, or used for epithelial cell isolation, as described below. All animal experiments were carried out in accordance with National Institutes of Health guidelines under protocols approved by the Institutional Animal Care and Use Committee at the University of Chicago.

Quantitative histopathology. Jejunal segments were fixed in neutral-buffered formalin and embedded in paraffin using established protocols. Well-oriented $5-\mu \mathrm{m}$ sections were stained with $\mathrm{H} \& \mathrm{E}$ and photographed using a light microscope equipped with a MicroPublisher 5.0 RTV camera (QImaging). For calculation of villous morphometry, the following measurements were obtained from 20 representative well-oriented villus-crypt units per group using Adobe Photoshop (Adobe Systems): distance from villus tip to the bottom of the adjacent crypts; distance from villus tip to the villus-crypt junction; and width of the villus at its midpoint. Ratios were calculated and compared using the 2-tailed Student's $t$ test. $P$ values less than 0.05 were considered statistically significant.

Intestinal permeability assay. Intestinal permeability and water flux were measured by adapting an in vivo assay previously used in rats (32). Seven- to 9-week-old female mice were fasted for 24 hours prior to each experiment. Anesthesia was induced 1 hour after treatment with anti-CD3 or vehicle with ketamine (75 mg/kg, intraperitoneal injection; Fort Dodge Animal Health) and xylazine $(25 \mathrm{mg} / \mathrm{kg}$, intraperitoneal injection; Lloyd Laboratories). Mice were injected intravenously with $250 \mu \mathrm{l} \mathrm{of} 1 \mathrm{mg} / \mathrm{ml}$ Alexa 488-conjugated BSA (Invitrogen Corp.). The abdomen was opened by a midline incision, and a $5-\mathrm{cm}$ loop of jejunum was cannulated at the proximal and distal ends with $0.76-\mathrm{mm}$ internal diameter polyethylene tubing. Flushing solution $(140 \mathrm{mM}$ $\mathrm{NaCl}, 10 \mathrm{mM}$ HEPES, $\mathrm{pH}$ 7.4) warmed to $37^{\circ} \mathrm{C}$ was first perfused through the jejunal loop at $1 \mathrm{ml} / \mathrm{min}$ for 10 minutes using a peristaltic pump (Bio-Rad Laboratories). This was followed by perfusion of $5 \mathrm{ml}$ test solution $(50 \mathrm{mM}$ $\mathrm{NaCl}, 5 \mathrm{mM}$ HEPES, $2 \mathrm{mM}$ sodium ferrocyanide, $2.5 \mathrm{mM} \mathrm{KCl}, 20 \mathrm{mM}$ glucose, $\mathrm{pH} 7.4$ ) in a recirculating manner at $1 \mathrm{ml} / \mathrm{min}$ for 3 hours, beginning 90 minutes after anti-CD 3 or vehicle treatment. The abdominal cavity was covered with moistened gauze, and body temperature, measured via rectal thermometer, was maintained at $37^{\circ} \mathrm{C}$ using a heating lamp. In experiments using isoosmotic perfusate, the perfusion solution consisted of $100 \mathrm{mM}$ $\mathrm{NaCl}, 10 \mathrm{mM}$ HEPES, $5 \mathrm{mM}$ sodium ferrocyanide, $5 \mathrm{mM} \mathrm{KCl}, 20 \mathrm{mM}$ glucose, $\mathrm{pH}$ 7.4. For experiments involving $\mathrm{Na}^{+}$-free perfusate, $\mathrm{N}$-methyl-D-glucamine-Cl was substituted for $\mathrm{NaCl}$. Alternatively, inhibitors were added to the perfusate when required, including PIK $(25-250 \mu \mathrm{M}$, as specified) (11), HOE694 (200 $\mu \mathrm{M})(77)$, S3226 (10 $\mu \mathrm{M})(78)$, niflumic acid (100 $\mu \mathrm{M})$, Y27632 $(10 \mu \mathrm{M})($ Calbiochem), Rac1 inhibitor NSC23766 (100 $\mu \mathrm{M})(45)$, and $20 \mu \mathrm{M}$ $\mathrm{AlF}_{4}^{-}$(Sigma-Aldrich). One-milliliter aliquots of test solution were removed at the beginning and end of the perfusion. After perfusion, the animal was sacrificed and the perfused jejunal segment excised and the length measured. The excised intestinal loop was then snap-frozen in OCT or used for epithelial cell isolation. Ferrocyanide concentration in the perfusate was measured using a previously described colorimetric assay (79). In preliminary experiments, $98 \%$ and $99 \%$ of perfused ferrocyanide was recovered from control and anti-CD3-treated animals after 2 hours of perfusion, respectively, confirming that this inert probe is not absorbed. Alexa 488-conjugated BSA concentration was measured using a microplate reader (Synergy HT; Bio-Tek) using an excitation wavelength of $485 \mathrm{~nm}$ and an emission wavelength of $528 \mathrm{~nm}$. Preliminary quantitative SDS-PAGE analysis showed that Alexa 488 fluorescence accurately represented the content of intact BSA in the luminal perfusate. Probe clearance was calculated as $C_{\text {probe }}=\left(C_{\mathrm{i}} V_{\mathrm{i}}-C_{\mathrm{f}} V_{\mathrm{f}}\right) /\left(C_{\text {avg }} T L\right)$; water flux was calculated as $\left(V_{\mathrm{i}}-V_{\mathrm{f}}\right) /(\mathrm{TL})$. In these equations, $C_{\mathrm{i}}$ represents the measured initial probe concentration; $C_{\mathrm{f}}$ represents the measured final probe concentration; $V_{\mathrm{i}}$ represents the measured initial perfusate volume; $V_{\mathrm{f}}$ was calculated as $V_{\mathrm{i}}\left([\text { ferrocyanide }]_{\mathrm{i}} /[\text { ferrocyanide }]_{\mathrm{f}}\right) ; C_{\text {avg }}$ was calculated as $\left(C_{\mathrm{i}}-C_{\mathrm{f}}\right) / \ln \left(C_{\mathrm{i}} / C_{\mathrm{f}}\right) ; T$ represents hours of perfusion; and $L$ represents the length of the perfused jejunal section in centimeters.

Immunofluorescence. Immunostaining was performed as previously described (49). Briefly, 5- $\mu \mathrm{m}$ frozen sections were collected on coated slides, fixed in $1 \%$ paraformaldehyde, and washed 3 times with PBS, and nonspecific binding was blocked with $1 \%$ normal goat serum in PBS. After incubation with affinity-purified anti-serine-19-phosphorylated MLC-specific rabbit antibody $(10 \mu \mathrm{g} / \mathrm{ml})(49)$, rabbit anti-occludin $(5 \mu \mathrm{g} / \mathrm{ml}$; Zymed Laboratories Inc.); rabbit anti-claudin-1, rabbit anti-claudin-5, and rabbit anti-JAM-A (10 $\mu \mathrm{g} / \mathrm{ml}$; Zymed Laboratories Inc.); rabbit anti-claudin-2 and rabbit anti-claudin-4 (5 $\mu \mathrm{g} / \mathrm{ml}$; Zymed Laboratories Inc.); or rabbit antiZO-1 antibody ( $2.5 \mu \mathrm{g} / \mathrm{ml}$; Zymed Laboratories Inc.), sections were washed and incubated with Alexa 594-conjugated goat anti-rabbit antisera, Alexa 488-conjugated phalloidin, and Hoechst 33342 (Invitrogen Corp.). Sections were imaged using a Leica DMLB epifluorescence microscope equipped with an 88000 filter set (Chroma Technology Corp.) and a Coolsnap HQ camera (Roper Scientific Inc.) controlled by MetaMorph 6 (Universal Imaging Corp.). Tight junction protein internalization was quantified by counting 500 villous enterocytes per group. The fractions of enterocytes with internalized occludin or JAM-A were compared using Student's $t$ test.

Epithelial isolation. We purified intestinal epithelial cells using a modification of a previously published method (80). Briefly, a fresh section of intestine was opened lengthwise and washed in $4{ }^{\circ} \mathrm{C} \mathrm{Ca}^{2+}$ - and $\mathrm{Mg}^{+}$-free HBSS (CMFHBSS). The tissue was then transferred to CMF-HBSS containing $10 \mathrm{mM}$ dithiothreitol and $50 \mathrm{nM}$ calyculin A (Calbiochem) and incubated for 30 minutes at $4^{\circ} \mathrm{C}$. After incubation, the tube was shaken briefly and the tissue transferred to a fresh tube containing CMF-HBSS with $1 \mathrm{mM}$ EDTA and $50 \mathrm{nM}$ calyculin A. After incubation at $4^{\circ} \mathrm{C}$ for 1 hour, epithelial cells were dislodged by vigorous shaking. Large pieces of tissue were removed from the tube and discarded, and epithelial cells were harvested by centrifugation at $500 \mathrm{~g}$ for 10 minutes.

Subcellular fractionation. Isolated intestinal epithelial cells were lysed in $2.5 \mathrm{ml}$ of Tris-buffered saline (TBS) containing 1\% Triton X-100. Two milliliters of this lysate was combined with $2 \mathrm{ml}$ of $80 \%$ sucrose (wt/vol) in TBS. The lysate in $40 \%$ sucrose was layered over a $1-\mathrm{ml}$ cushion of $50 \%$ 
sucrose. Sucrose layers were then added over the lysate as follows: $2 \mathrm{ml}$ of $35 \%$ sucrose, $2 \mathrm{ml}$ of $30 \%$ sucrose, $3 \mathrm{ml}$ of $15 \%$ sucrose, and $1 \mathrm{ml}$ of $5 \%$ sucrose. Sucrose gradients were then centrifuged at $280,000 \mathrm{~g}$ for 18 hours at $4{ }^{\circ} \mathrm{C}$. Fractions $(0.5 \mathrm{ml})$ were collected and analyzed for sucrose concentration, total protein using the DC Protein Assay (Bio-Rad Laboratories), and SDS-PAGE immunoblot.

SDS-PAGE and immunoblot analysis. Isolated intestinal epithelial cells were lysed in SDS sample buffer, sonicated on ice, and boiled for 5 minutes. These were separated by SDS-PAGE, transferred to PVDF, and probed using primary antibodies against total MLC, phosphorylated MLC (49), occludin (Zymed Laboratories Inc.), or caspase-3 (Cell Signaling Technology). Following incubation with appropriate HRP-conjugated secondary antibodies, signal was detected by chemiluminescence. Band intensity was determined by densitometry using MetaMorph 6 .

Analysis of cytokine transcription. Small pieces of intestine $\left(\sim 3 \mathrm{~mm}^{3}\right)$ were placed in TRIzol and sonicated briefly to disrupt the tissue. RNA was extracted with chloroform, precipitated with isopropanol, and resuspended in diethyl pyrocarbonate-treated water. RNA was further purified using a RNeasy mini kit (QIAGEN). cDNA was generated from $2 \mu \mathrm{g}$ RNA using Thermoscript reverse transcriptase (Invitrogen Corp.) and random hexamer primers in a $25-\mu l$ reaction. mRNA levels of TNF- $\alpha$ and IFN- $\gamma$ were determined by SYBR green real-time PCR using the MyiQ Real-Time PCR Detection System (Bio-Rad Laboratories) through 50 cycles. GAPDH was used as a reference. We used primers TCAAGTGGCATAGATGTGGAAGAA and TGGCTCTGCAGGATTTTCATG for detection of TNF- $\alpha$; CATCTTCTCAAAATTCGAGTGACAA and TGGGAGTAGACAAGGTACAACCC for detection of IFN- $\gamma$; and CTTCACCACCATGGAGAAGGC and GGCATGGACTGTGGTCATGAG for detection of GAPDH.

\section{Acknowledgments}

We are grateful to R.A. Anders and Y.-X. Fu for helpful discussions. HOE694 and S3226 were generously provided by J. Puenter (Aventis Pharma). D.R. Clayburgh is a predoctoral fellow of the NIH (The University of Chicago Medical Scientist Training Program T32 GM07281). This work was supported by grants from the NIH (R01DK61931 and R01DK68271 to J.R. Turner; R01DK54778 to T.A. Barrett; and R01DK48816 to L.L. Clarke) and the Crohn's and Colitis Foundation of America (to J.R. Turner). Support was also provided by the University of Chicago Digestive Disease Center (P30DK42086), the University of Chicago Cancer Center (P30CA14599), and the Northwestern Center for Drug Discovery and Chemical Biology.

Received for publication March 8, 2005, and accepted in revised form July 19, 2005.

Address correspondence to: Jerrold R. Turner, 5841 South Maryland, MC 1089, Chicago, Illinois 60637, USA. Phone: (773) 702-2433; Fax: (773) 834-5251; E-mail: jturner@bsd.uchicago.edu.
1. Yuhan, R., Koutsouris, A., Savkovic, S.D., and Hecht, G. 1997. Enteropathogenic Escherichia coliinduced myosin light chain phosphorylation alters intestinal epithelial permeability. Gastroenterology. 113:1873-1882.

2. Brown, G.R., et al. 1999. Tumor necrosis factor inhibitor ameliorates murine intestinal graft-versus-host disease. Gastroenterology. 116:593-601.

3. Breslin, N.P., et al. 2001. Intestinal permeability is increased in a proportion of spouses of patients with Crohn's disease. Am. J. Gastroenterol. 96:2934-2938

4. Wyatt, J., Vogelsang, H., Hubl, W., Waldhoer, T., and Lochs, H. 1993. Intestinal permeability and the prediction of relapse in Crohn's disease. Lancet. 341:1437-1439.

5. Yacyshyn, B.R., and Meddings, J.B. 1995. CD45RO expression on circulating CD19+ B cells in Crohn's disease correlates with intestinal permeability. Gastroenterology. 108:132-137.

6. Bjarnason, I., Marsh, M.N., Price, A., Levi, A.J., and Peters, T.J. 1985. Intestinal permeability in patients with coeliac disease and dermatitis herpetiformis. Gut. 26:1214-1219.

7. Hollander, D. 1988. Crohn's disease - a permeability disorder of the tight junction? Gut. 29:1621-1624.

8. Clayburgh, D.R., Shen, L., and Turner, J.R. 2004. A porous defense: the leaky epithelial barrier in intestinal disease. Lab. Invest. 84:282-291.

9. Hollander, D. 1992. The intestinal permeability barrier. A hypothesis as to its regulation and involvement in Crohn's disease. Scand. J. Gastroenterol. 27:721-726.

10. Vergnolle, N., et al. 2004. A role for proteinaseactivated receptor-1 in inflammatory bowel diseases. J. Clin. Invest. 114:1444-1456. doi:10.1172/ JCI200421689.

11. Zolotarevsky, Y., et al. 2002. A membrane-permeant peptide that inhibits MLC kinase restores barrier function in in vitro models of intestinal disease. Gastroenterology. 123:163-172.

12. Taylor, C.T., Dzus, A.L., and Colgan, S.P. 1998. Autocrine regulation of epithelial permeability by hypoxia: role for polarized release of tumor necrosis factor alpha. Gastroenterology. 114:657-668.

13. Suenaert, P., et al. 2002. Anti-tumor necrosis factor treatment restores the gut barrier in Crohn's disease. Am. J. Gastroenterol. 97:2000-2004.

14. Musch, M.W., et al. 2002. T cell activation causes diarrhea by increasing intestinal permeability and inhibiting epithelial $\mathrm{Na}^{+} / \mathrm{K}+-\mathrm{ATP}$ ase. J. Clin. Invest. 110:1739-1747. doi:10.1172/JCI200215695.

15. Bruewer, M., et al. 2003. Proinflammatory cytokines disrupt epithelial barrier function by apoptosis-independent mechanisms. J. Immunol. 171:6164-6172.

16. Mankertz, J., et al. 2000. Expression from the human occludin promoter is affected by tumor necrosis factor alpha and interferon gamma. J. Cell Sci. 113:2085-2090.

17. Youakim, A., and Ahdieh, M. 1999. Interferongamma decreases barrier function in T84 cells by reducing $Z \mathrm{ZO}-1$ levels and disrupting apical actin. Am. J. Physiol. 276:G1279-G1288.

18. Sugi, K., Musch, M.W., Field, M., and Chang, E.B. 2001. Inhibition of $\mathrm{Na}^{+}, \mathrm{K}+$-ATPase by interferon gamma down-regulates intestinal epithelial transport and barrier function. Gastroenterology. 120:1393-1403.

19. Gitter, A.H., Bendfeldt, K., Schulzke, J.D., and Fromm, M. 2000. Leaks in the epithelial barrier caused by spontaneous and TNF-alpha-induced single-cell apoptosis. FASEB J. 14:1749-1753.

20. Wang, F., et al. 2005. Interferon-gamma and tumor necrosis factor-alpha synergize to induce intestinal epithelial barrier dysfunction by up-regulating myosin light chain kinase expression. Am. J. Pathol. 166:409-419.

21. Ma, T.Y., Boivin, M.A., Ye, D., Pedram, A., and Said, H.M. 2005. Mechanism of TNF-alpha modulation of Caco-2 intestinal epithelial tight junction barrier: role of myosin light-chain kinase protein expression. Am. J. Physiol. Gastrointest. Liver Physiol. 288:G422-G430.

22. Turner, J.R., et al. 1997. Physiological regulation of epithelial tight junctions is associated with myosin light-chain phosphorylation. Am. J. Physiol.
273:C1378-C1385.

23. Scott, K.G., Meddings, J.B., Kirk, D.R., Lees-Miller, S.P., and Buret, A.G. 2002. Intestinal infection with Giardia spp. reduces epithelial barrier function in a myosin light chain kinase-dependent fashion. Gastroenterology. 123:1179-1190.

24. Clayburgh, D.R., et al. 2004. A differentiationdependent splice variant of myosin light chain kinase, MLCK1, regulates epithelial tight junction permeability. J. Biol. Chem. 279:55506-55513.

25. Chatenoud, L. 1988. Therapeutic use of the OKT3 anti-T cell monoclonal antibody: mode of action and side effects. Transplant. Proc. 20:79-83.

26. Charpentier, B., et al. 1992. Evidence that antihuman tumor necrosis factor monoclonal antibody prevents OKT3-induced acute syndrome. Transplantation. 54:997-1002.

27. Chatenoud, L., et al. 1990. In vivo cell activation following OKT3 administration. Systemic cytokine release and modulation by corticosteroids. Transplantation. 49:697-702.

28. Ferran, C., et al. 1990. Cytokine-related syndrome following injection of anti-CD3 monoclonal antibody: further evidence for transient in vivo $\mathrm{T}$ cell activation. Eur. J. Immunol. 20:509-515.

29. Matthys, P., et al. 1993. Modification of the antiCD3-induced cytokine release syndrome by antiinterferon-gamma or anti-interleukin-6 antibody treatment: protective effects and biphasic changes in blood cytokine levels. Eur. J. Immunol. 23:2209-2216.

30. Radojevic, N., et al. 1999. Characterization of enteric functional changes evoked by in vivo anti-CD3 T cell activation. Am. J. Physiol. 276:R715-R723.

31. Ferran, C., Bluestone, J., Bach, J.F., and Chatenoud, L. 1990. In vivo T lymphocyte activation induced in mice following the injection of anti-CD3 monoclonal antibody. Transplant. Proc. 22:1922-1923.

32. Sadowski, D.C., and Meddings, J.B. 1993. Luminal nutrients alter tight-junction permeability in the rat jejunum: an in vivo perfusion model. Can. J. Physiol. Pharmacol. 71:835-839.

33. Field, M. 2003. Intestinal ion transport and the pathophysiology of diarrhea. J. Clin. Invest. 
111:931-943. doi:10.1172/JCI200318326.

34. Ma, T., et al. 2002. Thiazolidinone CFTR inhibitor identified by high-throughput screening blocks cholera toxin-induced intestinal fluid secretion. J. Clin. Invest. 110:1651-1658. doi:10.1172/ JCI200216112.

35. Gawenis, L.R., et al. 2002. Intestinal $\mathrm{NaCl}$ transport in NHE2 and NHE3 knockout mice. Am. J. Physiol. Gastrointest. Liver Physiol. 282:G776-G784.

36. Zeiher, B.G., et al. 1995. A mouse model for the delta F508 allele of cystic fibrosis. J. Clin. Invest. 96:2051-2064.

37. Rahner, C., Mitic, L.L., and Anderson, J.M. 2001. Heterogeneity in expression and subcellular localization of claudins 2, 3, 4, and 5 in the rat liver, pancreas, and gut. Gastroenterology. 120:411-422.

38. Nusrat, A., et al. 2000. Tight junctions are membrane microdomains. J. Cell Sci. 113:1771-1781.

39. Wojciak-Stothard, B., Entwistle, A., Garg, R., and Ridley, A.J. 1998. Regulation of TNF-alpha-induced reorganization of the actin cytoskeleton and cellcell junctions by Rho, Rac, and Cdc42 in human endothelial cells. J. Cell. Physiol. 176:150-165.

40. Walsh, S.V., et al. 2001. Rho kinase regulates tight junction function and is necessary for tight junction assembly in polarized intestinal epithelia. Gastroenterology. 121:566-579.

41. Jou, T.S., Schneeberger, E.E., and Nelson, W.J. 1998. Structural and functional regulation of tight junctions by RhoA and Rac1 small GTPases. J. Cell Biol. 142:101-115.

42. Ries, J., Stein, J., Traynor-Kaplan, A.E., and Barrett, K.E. 1997. Dual role for AlF4(-)-sensitive G proteins in the function of T84 epithelial cells: transport and barrier effects. Am. J. Physiol. 272:C794-C803.

43. Hopkins, A.M., Walsh, S.V., Verkade, P., Boquet, P., and Nusrat, A. 2003. Constitutive activation of Rho proteins by CNF-1 influences tight junction structure and epithelial barrier function. J. Cell Sci. 116:725-742.

44. Uehata, M., et al. 1997. Calcium sensitization of smooth muscle mediated by a Rho-associated protein kinase in hypertension. Nature. 389:990-994.

45. Gao, Y., Dickerson, J.B., Guo, F., Zheng, J., and Zheng, Y. 2004. Rational design and characterization of a Rac GTPase-specific small molecule inhibitor. Proc. Natl. Acad. Sci. U. S. A. 101:7618-7623.

46. Steffen, A., et al. 2004. Sra-1 and Nap1 link Rac to actin assembly driving lamellipodia formation. EMBO J. 23:749-759.

47. Radhakrishna, H., Al-Awar, O., Khachikian, Z., and Donaldson, J.G. 1999. ARF6 requirement for Rac ruffling suggests a role for membrane trafficking in cortical actin rearrangements. J. Cell Sci. 112:855-866.

48. Madara, J.L. 1983. Increases in guinea pig small intestinal transepithelial resistance induced by osmotic loads are accompanied by rapid alterations in absorptive-cell tight-junction structure. J. Cell Biol. 97:125-136.

49. Berglund, J.J., Riegler, M., Zolotarevsky, Y., Wenzl,
E., and Turner,J.R. 2001. Regulation of human jejunal transmucosal resistance and MLC phosphorylation by $\mathrm{Na}(+)$-glucose cotransport. Am. J. Physiol. Gastrointest. Liver Physiol. 281:G1487-G1493.

50. Amano, M., et al. 1996. Phosphorylation and activation of myosin by Rho-associated kinase (Rhokinase). J. Biol. Chem. 271:20246-20249.

51. Kimura, K., et al. 1996. Regulation of myosin phosphatase by Rho and Rho-associated kinase (Rho-kinase). Science. 273:245-248.

52. Komatsu, S., and Ikebe, M. 2004. ZIP kinase is responsible for the phosphorylation of myosin II and necessary for cell motility in mammalian fibroblasts. J. Cell Biol. 165:243-254.

53. Yamashiro, S., et al. 2003. Citron kinase, a Rhodependent kinase, induces di-phosphorylation of regulatory light chain of myosin II. Mol. Biol. Cell. 14:1745-1756.

54. Kamm, K.E., and Stull, J.T. 2001. Dedicated myosin light chain kinases with diverse cellular functions. J. Biol. Chem. 276:4527-4530.

55. Verin, A.D., et al. 1998. Expression of a novel high molecular-weight myosin light chain kinase in endothelium. Am. J. Respir. Cell Mol. Biol. 19:758-766.

56. Wainwright, M.S., et al. 2003. Protein kinase involved in lung injury susceptibility: evidence from enzyme isoform genetic knockout and in vivo inhibitor treatment. Proc. Natl. Acad. Sci. U. S. A. 100:6233-6238.

57. Schwarze, S.R., Ho, A., Vocero-Akbani, A., and Dowdy, S.F. 1999. In vivo protein transduction: delivery of a biologically active protein into the mouse. Science. 285:1569-1572.

58. Owens, S.E., Graham, W.V., Siccardi, D., Turner, J.R., and Mrsny, R.J. 2005. A strategy to identify stable membrane-permeant peptide inhibitors of myosin light chain kinase. Pharm. Res. 22:703-709.

59. Rothbard, J.B., et al. 2000. Conjugation of arginine oligomers to cyclosporin A facilitates topical delivery and inhibition of inflammation. Nat. Med. 6:1253-1257.

60. Blue, E.K., et al. 2002. 220- and 130-kDa MLCKs have distinct tissue distributions and intracellular localization patterns. Am. J. Physiol. Cell Physiol. 282:C451-C460.

61. Verin, A.D., Gilbert-McClain, L.I., Patterson, C.E., and Garcia, J.G. 1998. Biochemical regulation of the nonmuscle myosin light chain kinase isoform in bovine endothelium. Am. J. Respir. Cell Mol. Biol. 19:767-776.

62. Merger, M., et al. 2002. Defining the roles of perforin, Fas/FasL, and tumour necrosis factor alpha in $\mathrm{T}$ cell induced mucosal damage in the mouse intestine. Gut. 51:155-163.

63. Ivanov, A.I., McCall, I.C., Parkos, C.A., and Nusrat, A. 2004. Role for actin filament turnover and a myosin II motor in cytoskeleton-driven disassembly of the epithelial apical junctional complex. Mol. Biol. Cell. 15:2639-2651.

64. Shen, L., and Turner, J.R. 2005. Actin depolymerization disrupts tight junctions via caveolae-medi- ated endocytosis. Mol. Biol. Cell. doi: $10.1091 / \mathrm{mbc}$. E04-12-1089.

65. Schmitz, H., et al. 1999. Tumor necrosis factoralpha (TNFalpha) regulates the epithelial barrier in the human intestinal cell line HT-29/B6. J. Cell Sci. 112:137-146.

66. Gitter, A.H., Wullstein, F., Fromm, M., and Schulzke, J.D. 2001. Epithelial barrier defects in ulcerative colitis: characterization and quantification by electrophysiological imaging. Gastroenterology. 121:1320-1328.

67. Somlyo, A.V., et al. 2004. Myosin light chain kinase knockout. J. Muscle Res. Cell Motil. 25:241-242.

68. Lukas, T.J., Mirzoeva, S., Slomczynska, U., and Watterson, D.M. 1999. Identification of novel classes of protein kinase inhibitors using combinatorial peptide chemistry based on functional genomics knowledge. J. Med. Chem. 42:910-919.

69. Bain, J., McLauchlan, H., Elliott, M., and Cohen, P. 2003. The specificities of protein kinase inhibitors: an update. Biochem. J. 371:199-204.

70. Blumenthal, D.K., and Stull, J.T. 1980. Activation of skeletal muscle myosin light chain kinase by calci$\mathrm{um}(2+)$ and calmodulin. Biochemistry. 19:5608-5614.

71. Gewirtz, A.T., et al. 2000. Salmonella typhimurium induces epithelial IL-8 expression via $\mathrm{Ca}(2+)$-mediated activation of the NF-кB pathway. J. Clin. Invest. 105:79-92.

72. Petrache, I., et al. 2003. Caspase-dependent cleavage of myosin light chain kinase (MLCK) is involved in TNF-alpha-mediated bovine pulmonary endothelial cell apoptosis. FASEB J. 17:407-416.

73. Lazar, V., and Garcia, J.G. 1999. A single human myosin light chain kinase gene (MLCK; MYLK) transcribes multiple nonmuscle isoforms. Genomics. 57:256-267.

74. Birukov, K.G., et al. 2001. Differential regulation of alternatively spliced endothelial cell myosin light chain kinase isoforms by p60(Src). J. Biol. Chem. 276:8567-8573.

75. Llor, X., et al. 1999. BRK/Sik expression in the gastrointestinal tract and in colon tumors. Clin. Cancer Res. 5:1767-1777.

76. Corredor, J., et al. 2003. Tumor necrosis factor regulates intestinal epithelial cell migration by receptor-dependent mechanisms. Am.J. Physiol. Cell Physiol. 284:C953-C961.

77. Schmid, A., Scholz, W., Lang, H.J., and Popp, R. 1992. $\mathrm{Na}+/ \mathrm{H}+$ exchange in porcine cerebral capillary endothelial cells is inhibited by a benzoylguanidine derivative. Biochem. Biophys. Res. Commun. 184:112-117.

78. Schwark, J.R., et al. 1998. S3226, a novel inhibitor of $\mathrm{Na}+/ \mathrm{H}+$ exchanger subtype 3 in various cell types. Pflugers Arch. 436:797-800.

79. Berliner, R.W., Kennedy, T.J., and Hilton, J.G. 1950. Renal clearance of ferrocyanide in the dog. Am. J. Physiol. 160:325-329.

80. Grossmann, J., et al. 1998. New isolation technique to study apoptosis in human intestinal epithelial cells. Am. J. Pathol. 153:53-62. 